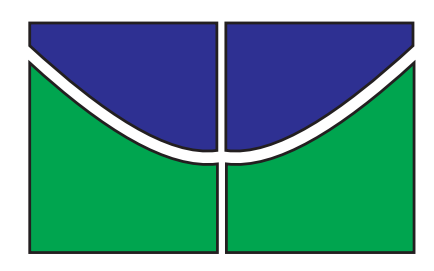

\author{
Universidade de Brasília \\ Instituto de Ciências Exatas \\ Departamento de Matemática
}

\title{
Ordem de Aparição na Sequência de Fibonacci: um Problema sobre Divisibilidade
}

Gustavo Candeia Costa

Brasília

2015 


\title{
Universidade de Brasília \\ Instituto de Ciências Exatas \\ Departamento de Matemática
}

\section{Ordem de Aparição na Sequência de Fibonacci: um Problema sobre Divisibilidade}

\author{
por \\ Gustavo Candeia Costa
}

Brasília

2015 
Universidade de Brasília

Instituto de Ciências Exatas

Departamento de Matemática

\section{Ordem de aparição na sequência de Fibonacci: Um problema} sobre divisibilidade.

\section{por \\ GUSTAVO CANDEIA COSTA*}

Dissertação apresentada ao Departamento de Matemática da Universidade de Brasília, como parte dos requisitos do "Programa" de Mestrado Profissional em Matemática em Rede Nacional - PROFMAT, para obtenção do grau de

\section{MESTRE}

Brasília, 03 de julho de 2015.

Comissão Examinadora:

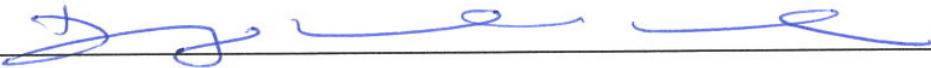

Prof. Dr. Diego Marques Ferreira - MAT/UnB (Orientador)
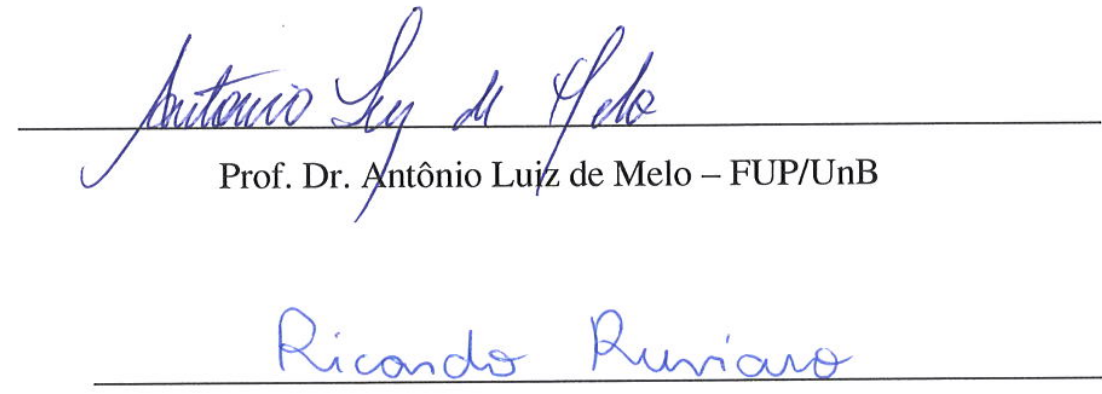

Prof. Dr. Ricardo Ruviaro - MAT/UnB 
Ficha catalográfica elaborada automaticamente, com os dados fornecidos pelo(a) autor(a)

Ordem de aparição na sequência de Fibonacci: um

problema sobre divisibilidade / Gustavo Costa

Candeia; orientador Diego Marques. -- Brasília, 2015. $81 \mathrm{p}$.

Dissertação (Mestrado - Mestrado em Matemática) -Universidade de Brasília, 2015.

1. Ordem de aparição. 2. Números de Fibonacci. 3. Números de Lucas. 4. Divisibilidade. I. Marques, Diego, orient. II. Título. 
À minha esposa e aos meus filhos, meus alicerces. 


\section{AGRADECIMENTOS}

A Deus, Senhor e Mestre da minha vida, por Se mostrar a esse jovem em um momento tão crítico de busca e incredulidade. Por colocar em minha mente e em meu coração a certeza da Sua presença viva, de maneira que eu enxergasse a necessidade de viver em prol de um bem maior.

À minha família, em especial aos meus pais. Eles foram essenciais na formação do meu caráter. Com a minha mãe aprendi o valor da educação e com o meu pai aprendi que os sonhos não têm limites! Somos capazes de realizar aquilo que queremos.

Aos professores: Carlos, Adail de Castro Cavalheiro, Aline Gomes da Silva Pinto, Ary Vasconcelos Medino, Daniele da Silva Baratela Martins Neto, Lineu da Costa Araújo Neto, Lucas Conque Seco Ferreira, Mauro Luiz Rabelo, Raquel Carneiro Dörr, Ricardo Ruviaro e Rui Seimetz pela contribuição na minha formação Matemática.

Ao professor orientador e amigo - Diego Marques - pelo entusiasmo com que realiza suas aulas e pesquisas. Pelas extraordinárias contribuições à Matemática. Por fazer despertar em seus alunos a vontade de ir além. E pela imensa ajuda na realização desse texto.

Aos colegas de curso: Ana Paula, Daniel, Douglas, Emerson, Emmanuel, Fred, Marco, Maryna, Ricardo, Ronald e Ulysses por compartilhar várias horas de estudo durante todo o mestrado.

Aos demais colegas de turma e aos envolvidos com o PROFMAT por engrandecer esse mestrado profissionalizante. 
À mestranda Anna Carolina Lafetá por dedicar um pouco do seu tempo à leitura desse trabalho e pelas excelentes observações feitas.

À minha esposa Fabiana por ser exemplo de companheirismo. Por estar ao meu lado em cada decisão tomada. Por me incentivar. Por fazer de mim uma pessoa melhor. Por construir uma família e uma vida tão maravilhosa comigo. Por entender as minhas ausências durante esses dois anos e meio de estudos. Ela que se mostrou companheira e amiga durante o nosso tempo juntos, não deixou de fazê-lo nas horas mais difíceis dessa caminhada. Cada momento longe do seio familiar foi suportado com a certeza de uma união em Cristo, Ele que nos dá força e nos conduz pelos Seus caminhos.

Fabiana, eu te amo! Não conseguiria chegar onde cheguei sem você! 
"Quem procura a verdade procura a Deus, ainda que não o saiba.” (Edith Stein) 


\section{RESUMO}

Seja $\left(F_{n}\right)_{n \geq 0}$ a sequência de Fibonacci e $z(n)$ a ordem de aparição nessa sequência definida como o menor $k \in \mathbb{N}$ tal que $n$ divide $F_{k}$. Nesse trabalho, discutiremos algumas propriedades dessa função. O principal objetivo é provar que existem infinitas soluções para a equação $z(n)=z(n+2)$ e exibir fórmulas fechadas para $z\left(F_{m} \pm 1\right)$. Mas, antes disso, detalharemos propriedades dos números de Fibonacci e números de Lucas.

\section{Palavras-chave}

Números de Fibonacci, números de Lucas e ordem de aparição. 


\section{ABSTRACT}

Let $\left(F_{n}\right)_{n \geq 0}$ be the Fibonacci sequence and let $z(n)$ be the order of appearance in this sequence which is defined as the smallest $k \in \mathbb{N}$ such that $n$ divides $F_{k}$. In this work, we shall discuss some properties of this function. The main goal is to prove the existence of infinitely many solutions to the equation $z(n)=z(n+2)$ as well as to exhibit closed formulas for $z\left(F_{m} \pm 1\right)$. At first, we shall describe the properties of Fibonacci and Lucas numbers.

\section{Keywords}

Numbers of Fibonacci, numbers of Lucas and order of appearance. 


\section{LISTA DE FIGURAS}

1.1 Retângulos $1 \times 4 \ldots \ldots \ldots \ldots \ldots$. . . . . . . . . . . . .

1.2 Espiral de Fibonacci . . . . . . . . . . . . . . . . 11

1.3 Soma das diagonais do triângulo de Pascal . . . . . . . . . . . . . . 12

1.4 Intervalos encaixantes . . . . . . . . . . . . . . . 17

4.1 Tijolo de Fibonacci . . . . . . . . . . . . . . 58

A.1 Esfera circunscrita ao tijolo de Fibonacci . . . . . . . . . . . . . . 64 


\section{SUMÁRIO}

$\begin{array}{ll}\text { Introdução } & 1\end{array}$

1 Números de Fibonacci e Números de Lucas 3

1.1 Sequência de Fibonacci . . . . . . . . . . . . . . . . 3

1.2 Somas de números da sequência de Fibonacci . . . . . . . . . . . 8

1.3 Fibonacci e algumas relações interessantes . . . . . . . . . . . . . . 12

1.4 Números de Lucas . . . . . . . . . . . . . . . . . . 18

2 Divisibilidade e Números de Fibonacci e de Lucas $\quad 23$

2.1 Resultados clássicos sobre divisibilidade . . . . . . . . . . . . . 23

2.2 Símbolo de Legendre e resultados usando congruências módulo $p$ primo. 29

2.3 Teoremas auxiliares . . . . . . . . . . . . . . . . 32

3 Ordem de Aparição na Sequência de Fibonacci 37

3.1 Ordem de aparição na sequência de Fibonacci . . . . . . . . . . . . 37

3.2 Infinitas soluções para $z(n)=z(n+2)$ e fórmulas fechadas para $z\left(F_{m} \pm 1\right) 44$

4 Aplicações ao Ensino Médio $\quad 53$

4.1 Pequeno teorema de Fermat . . . . . . . . . . . . . . 53

4.2 Sugestão de atividades e problemas . . . . . . . . . . . 56

$\begin{array}{ll}\text { Considerações finais } & 60\end{array}$ 
A Problemas Aplicáveis ao Ensino Médio

Referências 


\section{INTRODUÇÃO}

Este trabalho apresentará um estudo sobre a ordem de aparição na sequência de Fibonacci. Para atingir esse objetivo, será necessário conhecer os números de Fibonacci e algumas de suas propriedades.

Dada a sequência de Fibonacci $\left(F_{n}\right)_{n \geq 0}$ definida por $F_{n+2}=F_{n+1}+F_{n}$, para $n \geq 0$, onde $F_{0}=0$ e $F_{1}=1$, demonstraremos alguns resultados clássicos da literatura e outros que servirão de suporte para a parte central do texto, que é a ordem de aparição na sequência de Fibonacci.

Seja $F_{n}$ o $n$-ésimo número de Fibonacci. A ordem de aparição $z(n)$ de um número natural $n$ na sequência de Fibonacci é definida como o menor número natural $k$ tal que $n$ divide $F_{k}$.

Com isso algumas perguntas surgem naturalmente. Por exemplo, $z(n)$ está sempre definida? Existem fórmulas fechadas para $z(n)$ ? Quais as condições para que $z(n)$ seja igual a $z(n+1)$ e para $z(n)=z(n+2)$ ? Quando $z(n)=2 n$ ? Será que $z\left(F_{n}\right)$ coincide com a posição de $F_{n}$ ? Desses questionamentos, o último é consequência imediata da definição de ordem de aparição na sequência de Fibonacci.

Demonstraremos alguns resultados sobre ordem de aparição, como por exemplo: se $m \mid F_{n}$, então $z(m) \mid n$, e $z\left(F_{m} \pm 1\right)>m=z\left(F_{m}\right)$, para todo $m \geq 5$. Mostraremos que existem infinitas soluções para $z(n)=z(n+2)$.

Forneceremos fórmulas explícitas para $z\left(F_{m} \pm 1\right)$ dependendo da classe de restos de $m$ módulo 4. Em particular, $z\left(F_{m} \pm 1\right) \geq\left(m^{2} / 2\right)-2$, para $m \equiv 0(\bmod 4)$.

Em geral, o primeiro capítulo tratará da introdução à sequência de Fibonacci. 
Além de relembrarmos o famoso problemas dos coelhos, vamos dar exemplos abstratos onde a resposta é dada por números de Fibonacci. Também no primeiro capítulo, vamos demonstrar, usando o princípio de indução, alguns resultados sobre somas de números de Fibonacci.

Encerrando essa primeira parte do trabalho, vamos relacionar a sequência de Fibonacci a alguns tópicos interessantes ligados a ela, como por exemplo, triplas pitagóricas, triângulo de Pascal e razão áurea.

O segundo capítulo apresentará resultados e propriedades clássicas envolvendo conceitos de divisibilidade relacionados aos números de Fibonacci e de Lucas. Esses resultados darão suporte para as demonstrações que serão feitas no capítulo seguinte. No final do segundo capítulo, comentaremos sobre as somas de potências de números de Fibonacci consecutivos.

No terceiro capítulo, trataremos da ordem de aparição na sequência de Fibonacci. Apresentaremos uma tabela com a ordem de aparição dos 100 primeiros números naturais. Essa tabela pode ser usada para atividades onde o objetivo é fazer inferências e conjecturas sobre determinados padrões numéricos. Vamos mostrar alguns resultados envolvendo os números de Fibonacci, os números de Lucas e a ordem de aparição de um número natural. No ápice do texto, demonstraremos que há infinitas soluções para $z(n)=z(n+2)$ e caracterizaremos $z\left(F_{m} \pm 1\right)$, dependendo do resto da divisão de $m$ por 4 .

No quarto e último capítulo, de maneira geral, faremos uma breve explanação sobre a aplicabilidade de alguns dos conceitos sobre divisibilidade contidos no trabalho às séries finais do Ensino Médio. Enfatizando, dessa forma, a importância da teoria elementar dos números para a formação básica do estudante. 


\section{CAPÍTULO 1}

\section{NÚMEROS DE FIBONACCI E NÚMEROS DE LUCAS}

\subsection{Sequência de Fibonacci}

Leonardo de Pisa nasceu na Itália por volta de 1175 e ficou conhecido como Leonardo Fibonacci, ou simplesmente Fibonacci (filho de Bonacci), uma vez que o nome do seu pai era Guilielmo Bonacci. Na sua obra Liber abacci, ou livro do ábaco, há o registro do problema dos coelhos, o qual geraria uma das sequências numéricas mais famosas da humanidade. De acordo com [5], Fibonacci foi um dos melhores matemáticos do período medieval, publicando, além do Liber abacci, os trabalhos Practica Geometriae, em 1220, sobre geometria e trigonometria e Liber quadratorum, em 1225, sobre análise indeterminada.

O problema dos coelhos era praticamente o seguinte: "Um homem pôs um casal de coelhos em um lugar cercado por todos os lados por um muro. Quantos pares de coelhos podem ser gerados a partir desse par em um ano se, supostamente, todo mês cada casal de coelhos gera um novo casal, que é fértil a partir do segundo mês?"

Uma suposição adicional é que não há mortes de coelhos no período considerado.

Vale ressaltar que Fibonacci não fez um experimento real sobre reprodução de 
coelhos. Pelo contrário, ele propôs uma questão matemática supostamente sem a pretensão de ligá-la a outros campos do conhecimento.

Ao analisar o problema, percebe-se que no momento inicial há um par de coelhos jovens e inférteis. Após o primeiro mês, quando o casal se torna fértil, ele pode reproduzir, mas ainda só existe um casal.

No segundo mês haverá dois casais, um adulto e o outro jovem. No terceiro mês, o casal inicial terá gerado mais um casal totalizando três casais.

No quarto mês, o casal matriz gerará outro casal. O primeiro casal de coelhos gerado também contribuirá com outro casal de coelhos, de modo que já são cinco casais.

Continuando esse processo, onde a partir do início do terceiro mês a quantidade de casais de coelhos é igual a soma da quantidade de casais dos dois meses imediatamente anteriores, surge a sequência numérica conhecida como sequência de Fibonacci ${ }^{1}$.

Assim os primeiros números da sequência de Fibonacci são:

$1,1,2,3,5,8,13,21,34,55,89,144,233,377,610,987,1597,2584, \ldots$

Dessa forma, após um ano, são gerados 232 pares de coelhos a partir do casal de coelhos inicial.

Sem o aspecto histórico ou a contextualização, podemos definir a sequência de Fibonacci da seguinte forma.

Definição 1.1. A sequência de Fibonacci $\left(F_{n}\right)_{n \geq 0}$ é definida por $F_{n+2}=F_{n+1}+F_{n}$, para $n \geq 0$, onde $F_{0}=0$ e $F_{1}=1$.

Essa sequência numérica tem muitas propriedades interessantes e algumas delas serão abordadas nesse texto. Para informações mais avançadas, o leitor pode consultar [1] ou [23]. Sequências como a de Fibonacci são chamadas de recorrentes ou recursivas.

Analisando a recorrência linear de segunda ordem homogênea com coeficientes constantes $\left(F_{n+2}=F_{n+1}+F_{n}\right.$, para $n \geq 0$, onde $F_{0}=0$ e $\left.F_{1}=1\right)$, é possível determinar uma expressão para o termo geral dessa recorrência. Essa expressão é conhecida como fórmula de Binet.

Observe que a equação característica da recorrência acima é $\varphi^{2}-\varphi-1=0$ (ver [11]), cujas raízes são $\alpha=(1+\sqrt{5}) / 2$ ou $\beta=(1-\sqrt{5}) / 2$.

Sabemos que se $r_{1}$ e $r_{2}$ são raízes distintas de $r^{2}+p r+q=0, p, q \in \mathbb{R}$, então $X_{n}=c_{1} r_{1}^{n}+c_{2} r_{2}^{n}$ é solução da recorrência $X_{n+2}+p X_{n+1}+q X_{n}=0$, quaisquer que sejam os valores das constantes $c_{1}$ e $c_{2}$.

\footnotetext{
${ }^{1} \mathrm{O}$ nome sequência de Fibonacci foi dado pelo matemático Édouard Lucas, 1842-1891.
} 
De fato, sejam $Y_{n}=r_{1}^{n}, Z_{n}=r_{2}^{n}$ e $X_{n}=c_{1} Y_{n}+c_{2} Z_{n}$. Assim

$$
\begin{aligned}
X_{n+2}+p X_{n+1}+q X_{n} & =\left(c_{1} Y_{n+2}+c_{2} Z_{n+2}\right)+p\left(c_{1} Y_{n+1}+c_{2} Z_{n+1}\right)+q\left(c_{1} Y_{n}+c_{2} Z_{n}\right) \\
& =c_{1}\left(Y_{n+2}+p Y_{n+1}+q Y_{n}\right)+c_{2}\left(Z_{n+2}+p Z_{n+1}+q Z_{n}\right) \\
& =c_{1}(0)+c_{2}(0)=0 .
\end{aligned}
$$

Dessa forma, o termo geral da sequência de Fibonacci é dado por $F_{n}=c_{1} \alpha^{n}+c_{2} \beta^{n}$, $\operatorname{com} F_{0}=0, F_{1}=1, c_{1}, c_{2} \in \mathbb{R}$ e $\alpha$ e $\beta$ raízes da equação $\varphi^{2}-\varphi-1=0$.

Logo,

$$
\left\{\begin{array} { l } 
{ F _ { 0 } = 0 = c _ { 1 } \alpha ^ { 0 } + c _ { 2 } \beta ^ { 0 } = c _ { 1 } + c _ { 2 } } \\
{ F _ { 1 } = 1 = c _ { 1 } \alpha ^ { 1 } + c _ { 2 } \beta ^ { 1 } = c _ { 1 } \alpha + c _ { 2 } \beta }
\end{array} \Rightarrow \left\{\begin{array}{l}
c_{1}+c_{2}=0 \\
c_{1} \alpha+c_{2} \beta=1
\end{array} .\right.\right.
$$

O que implica

$$
c_{1}=-c_{2} \Rightarrow-c_{2} \alpha+c_{2} \beta=1 \Rightarrow c_{2}(-\alpha+\beta)=1 \Rightarrow c_{2}=-1 / \sqrt{5} \text { e } c_{1}=1 / \sqrt{5} \text {. }
$$

Portanto,

$$
F_{n}=\frac{\alpha^{n}-\beta^{n}}{\alpha-\beta} .
$$

É, no mínimo, bastante curioso o fato de que para qualquer inteiro $n$ não negativo $F_{n}=\left(\alpha^{n}-\beta^{n}\right) / \sqrt{5}$ também seja inteiro. Por exemplo $F_{7}=13, F_{67}=$ 44.945.570.212.853 e $F_{127}=155.576 .970 .220 .531 .065 .681 .649 .693$.

Esses três números exemplificam a periodicidade dos dígitos das unidades dos números de Fibonacci, ou seja, $F_{k+60} \equiv F_{k}(\bmod 10)$ para $k \geq 0$. Essa demonstração pode ser feita por indução. Vamos mostrar esse fato no segundo capítulo, na Proposição 2.11.

Outro fato interessante é que, para $p$ primo $(p<300), F_{p}$ é primo ou um produto de primos distintos ${ }^{2}$, ou seja, $F_{p}$ é livre de quadrados. Os 10 primeiros números primos $F_{p}$ são os seguintes: $F_{3}, F_{5}, F_{7}, F_{11}, F_{13}, F_{17}, F_{23}, F_{29}, F_{43}, F_{47}$.

Observe que $F_{19}=4181=37 \times 113, F_{31}=1346269=557 \times 2417$, e $F_{59}=$ $956722026041=353 \times 2710260697$ são números compostos. Também são compostos os números $F_{37}, F_{41}, F_{53}$, apesar de 19,31,37,41,53 e 59 serem primos.

Nesse sentido é possível provar que se $k=m \times n,(k>4), 1<m, n<k$, é composto, então $F_{k}$ também é composto. A Proposição 2.6 mostrará esse fato. Dessa forma,

\footnotetext{
${ }^{2}$ Esses fatos podem ser vistos em http://www.maths.surrey.ac.uk/hosted-
} sites/R.Knott/Fibonacci/fibtable.html 
$p$ primo é uma condição necessária, porém não suficiente, para $F_{p}$ ser primo. Até o momento da realização desse trabalho, ainda estava em aberto a questão da existência de infinitos primos na sequência de Fibonacci.

Entre os primeiros 300 números de Fibonacci o maior primo encontrado é o $F_{137}=$ 19.134.702.400.093.278.081.449.423.917. Também são primos os seguintes números de Fibonacci: $F_{359}, F_{431}, F_{433}$ e $F_{449}$.

Em muitos livros, por exemplo em [1] e [5], o termo geral da sequência de Fibonacci, $F_{n}=\left(\alpha^{n}-\beta^{n}\right) / \sqrt{5}$, é apresentado como a fórmula de Binet ${ }^{3}$. Nesses livros, no lugar da construção da expressão (1.1), usando o conhecimento sobre recorrências, é apresentada uma demonstração por indução como a seguinte.

Proposição 1.2. O n-ésimo número de Fibonacci é dado por $F_{n}=\left(\alpha^{n}-\beta^{n}\right) / \sqrt{5}=$ $\left(\alpha^{n}-\beta^{n}\right) /(\alpha-\beta)$, onde $\alpha=(1+\sqrt{5}) / 2$ e $\beta=(1-\sqrt{5}) / 2$ são as raízes da equação $\varphi^{2}-\varphi-1=0$.

Demonstração. Observe que a igualdade $F_{n}=\left(\alpha^{n}-\beta^{n}\right) / \sqrt{5}$ é válida para $n=0$ e $n=1$, pois $F_{0}=\left(\alpha^{0}-\beta^{0}\right) / \sqrt{5}=0$ e $F_{1}=\left(\alpha^{1}-\beta^{1}\right) / \sqrt{5}=1$.

Agora, vamos supor que, para todo inteiro $1<k \leq n+1$, a expressão $F_{k}=$ $\left(\alpha^{k}-\beta^{k}\right) / \sqrt{5}$ seja válida. Queremos mostrar que (1.1) se verifica para $k=n+2$, ou seja, $F_{n+2}=\left(\alpha^{n+2}-\beta^{n+2}\right) / \sqrt{5}$.

Pela hipótese de indução, temos: $F_{n}=\left(\alpha^{n}-\beta^{n}\right) / \sqrt{5}$ e $F_{n+1}=\left(\alpha^{n+1}-\beta^{n+1}\right) / \sqrt{5}$. Substituindo $F_{n}$ e $F_{n+1}$ na expressão para $F_{n+2}$, dada na definição da sequência de Fibonacci, temos:

$$
\begin{aligned}
F_{n+2} & =F_{n+1}+F_{n} \\
& =\frac{1}{\sqrt{5}}\left(\alpha^{n+1}-\beta^{n+1}\right)+\frac{1}{\sqrt{5}}\left(\alpha^{n}-\beta^{n}\right) \\
& =\frac{1}{\sqrt{5}}\left(\alpha^{n}\right)(\alpha+1)-\frac{1}{\sqrt{5}}\left(\beta^{n}\right)(\beta+1) \\
& =\frac{1}{\sqrt{5}}\left(\alpha^{n}\right)\left(\frac{3+\sqrt{5}}{2}\right)-\frac{1}{\sqrt{5}}\left(\beta^{n}\right)\left(\frac{3-\sqrt{5}}{2}\right) \\
& =\frac{1}{\sqrt{5}}\left(\alpha^{n}\right) \alpha^{2}-\frac{1}{\sqrt{5}}\left(\beta^{n}\right) \beta^{2} \\
& =\frac{\alpha^{n+2}-\beta^{n+2}}{\alpha-\beta} .
\end{aligned}
$$

Observe que usamos as seguintes igualdades: $(3+\sqrt{5}) / 2=\alpha^{2}$ e $(3-\sqrt{5}) / 2=\beta^{2}$.

\footnotetext{
${ }^{3}$ Fórmula de Binet - fórmula não recursiva para os números de Fibonacci.
} 
Portanto, a expressão (1.1) se verifica para todo inteiro não negativo $n$.

É bastante comum, nos livros que abordam a sequência de Fibonacci, exemplos da vida real onde se encontram termos da referida sequência ligados a observações da natureza. Por exemplo, em [5] e [2] existem excelentes informações. Também é bastante comum explorar a sequência de Fibonacci simultaneamente à razão áurea. Todavia, nesse texto, vamos em um sentido diferente. A seguir, citaremos dois exemplos abstratos onde surgem os números de Fibonacci.

Exemplo 1.3. Considere um retângulo $1 \times n$, o qual pode ser preenchido por dois tipos de retângulos menores, $1 \times 1$ e $1 \times 2$. De quantas maneiras podemos fazer isso?

Solução. Se $n=1$, só há uma maneira de cobrir o retângulo. Se $n=2$, há duas maneiras. Se $n=3$, então existem três maneiras distintas de preencher o retângulo. Se $n=4$, há 5 modos distintos, a saber:
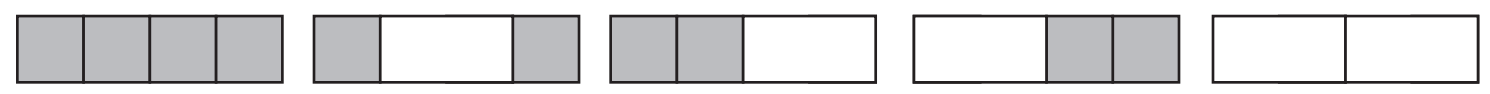

Figura 1.1: Retângulos $1 \times 4$

De maneira geral, seja $X_{n}$ o número de maneiras distintas de preencher o retângulo $1 \times n$. Assim, $X_{1}=1, X_{2}=2, X_{3}=3, X_{4}=5, X_{5}=8, \ldots$

Observe que para cobrir o retângulo $1 \times n$, ou começamos com um retângulo $1 \times 1$, faltando $(n-1)$ casas para serem preenchidas, o que pode ser feito de $X_{n-1}$ maneiras, ou começamos com um retângulo $1 \times 2$, restando $(n-2)$ casas para serem preenchidas, o que pode ser feito de $X_{n-2}$ modos.

Portanto, o número de maneiras distintas de cobrir o retângulo $1 \times n$ é $X_{n}=$ $X_{n-1}+X_{n-2}, \operatorname{com} X_{1}=1$ e $X_{2}=2$.

A sequência numérica, que é solução para o problema com $n=1,2,3, \ldots$ pode ser vista como a de Fibonacci, porém com um deslocamento. Desse modo, a quantidade de maneiras do retângulo $1 \times n$ ser preenchido, de acordo com as condições dadas, é igual a

$$
X_{n}=\frac{\left(\frac{1+\sqrt{5}}{2}\right)^{n+1}-\left(\frac{1-\sqrt{5}}{2}\right)^{n+1}}{\sqrt{5}}=\frac{\alpha^{n+1}-\beta^{n+1}}{\alpha-\beta} .
$$


Exemplo 1.4. Há n lâmpadas enfileiradas em uma sala. Quantas configurações existem se não puder haver duas lâmpadas adjacentes ligadas simultaneamente?

Solução. Seja $A_{n}$ o número de configurações para $n$ lâmpadas.

Vamos contar separadamente os casos onde a primeira lâmpada está desligada e posteriormente somar à quantidade de casos onde a primeira lâmpada está ligada e assim obter o total $A_{n}$.

Dessa forma, temos:

$$
A_{n}=\underbrace{A_{n-1}}_{\text {Primeira lâmpada desligada }}+\underbrace{A_{n-2}}_{\text {Primeira lâmpada ligada }} .
$$

Observe que se a primeira lâmpada está ligada, então necessariamente a lâmpada adjacente deve estar desligada e com isso há $A_{n-2}$ configurações distintas para o caso em que a primeira lâmpada está ligada.

Dessa forma, $A_{1}=2, A_{2}=3, A_{3}=5$ e, a cada lâmpada acrescentada na sala, a partir da terceira, o total de configurações é dado pela soma das duas quantidades imediatamente anteriores.

Analogamente ao exemplo anterior, surge nas respostas para as configurações com $n=1,2,3, \ldots$ lâmpadas a sequência de Fibonacci com um deslocamento de duas posições, ou seja, $A_{n}=F_{n+2}$. Por exemplo, $A_{10}=F_{12}=144$. Desse modo, o número de configurações para $n$ lâmpadas é igual a

$$
A_{n}=\frac{1}{\sqrt{5}}\left[\left(\frac{1+\sqrt{5}}{2}\right)^{n+2}-\left(\frac{1-\sqrt{5}}{2}\right)^{n+2}\right]=\frac{\alpha^{n+2}-\beta^{n+2}}{\alpha-\beta} .
$$

\subsection{Somas de números da sequência de Fibonacci}

Ao observar a sequência de Fibonacci, alguns padrões parecem se repetir. Observando esses padrões, vamos destacar algumas relações entre a soma de determinados números e posteriormente demonstrá-las.

Por exemplo, a soma dos $n$ primeiros números de Fibonacci é $F_{n+2}-1$, isto é,

$$
F_{1}+F_{2}+F_{3}+\cdots+F_{n}=F_{n+2}-1 \text {. }
$$

A soma dos $n$ primeiros números de Fibonacci de índice par é igual ao número de Fibonacci seguinte menos uma unidade, ou seja,

$$
F_{2}+F_{4}+F_{6}+\cdots+F_{2 n}=F_{2 n+1}-1 .
$$


A soma dos $n$ primeiros números de Fibonacci de índice ímpar é a seguinte:

$$
F_{1}+F_{3}+F_{5}+\cdots+F_{2 n-1}=F_{2 n} .
$$

A soma dos quadrados dos $n$ primeiros números de Fibonacci é

$$
F_{1}^{2}+F_{2}^{2}+F_{3}^{2}+\cdots+F_{n}^{2}=F_{n} F_{n+1}
$$

Além disso, o padrão dos primeiros números da sequência de Fibonacci sugere que dois números de Fibonacci consecutivos são primos entre si, ou seja, $\operatorname{mdc}\left(F_{n}, F_{n+1}\right)=1$, para todo $n \in \mathbb{N}$.

Quando não houver prejuízo para o entendimento vamos denotar o máximo divisor comum de dois números $a$ e $b$ por $(a, b)$.

Vamos mostrar esses resultados usando o princípio de indução.

Proposição 1.5. Quaisquer dois números de Fibonacci consecutivos são primos entre si, ou seja, $\left(F_{n}, F_{n+1}\right)=1, \forall n \in \mathbb{N}$.

Demonstração. Observe que $\left(F_{1}, F_{2}\right)=1$ e $\left(F_{2}, F_{3}\right)=1$. Suponha que $\left(F_{n}, F_{n+1}\right)=1$. Queremos mostrar que $\left(F_{n+1}, F_{n+2}\right)=1$. Sabemos que $F_{n+2}=F_{n+1}+F_{n}$ e que se $(a, b-n a)$ está definido, $a, b, n \in \mathbb{Z}$, então $(a, b)=(a, b-n a)$, ver [7]. Assim,

$$
\left(F_{n+1}, F_{n+2}\right)=\left(F_{n+1}, F_{n+1}+F_{n}\right)=\left(F_{n+1}, F_{n+1}+F_{n}-F_{n+1}\right)=\left(F_{n+1}, F_{n}\right) .
$$

Como, por hipótese de indução, $\left(F_{n+1}, F_{n}\right)=1$, segue que $\left(F_{n+1}, F_{n+2}\right)=1$.

Portanto, pelo princípio de indução finita, $\left(F_{n}, F_{n+1}\right)=1, \forall n \in \mathbb{N}$.

Proposição 1.6. $F_{1}+F_{2}+F_{3}+\cdots+F_{n}=F_{n+2}-1$ para todo $n \in \mathbb{N}$.

Demonstração. Observe que $F_{1}=F_{1+2}-1$, isto é, $1=F_{3}-1=1$ e que $F_{1}+F_{2}=$ $F_{2+2}-1=F_{4}-1=2$. Agora, supondo que $F_{1}+F_{2}+F_{3}+\cdots+F_{n}=F_{n+2}-1$ seja verdadeira, queremos provar que $F_{1}+F_{2}+F_{3}+\cdots+F_{n}+F_{n+1}=F_{n+3}-1$ também é verdadeira.

Por hipótese de indução, temos $F_{1}+F_{2}+F_{3}+\cdots+F_{n}=F_{n+2}-1$. Assim, somando $F_{n+1}$ a ambos os lados da igualdade anterior, temos:

$$
F_{1}+F_{2}+\cdots+F_{n}+F_{n+1}=F_{n+1}+F_{n+2}-1 \text {. }
$$

Porém, $F_{n+1}+F_{n+2}-1=F_{n+3}-1$. Dessa forma,

$$
F_{1}+F_{2}+F_{3}+F_{n}+\cdots+F_{n+1}=F_{n+3}-1 .
$$


Portanto, pelo princípio de indução, $F_{1}+F_{2}+F_{3}+F_{4}+\cdots+F_{n}=F_{n+2}-1$ é válida para todo $n \in \mathbb{N}$.

Proposição 1.7. $F_{2}+F_{4}+F_{6}+\cdots+F_{2 n}=F_{2 n+1}-1$, para todo $n \in \mathbb{N}$.

Demonstração. Para a base de indução, temos $F_{2}=F_{2+1}-1=1$ e $F_{2}+F_{4}=F_{5}-1=4$.

Suponha que $F_{2}+F_{4}+F_{6}+\cdots+F_{2 n}=F_{2 n+1}-1$ para um certo $n$ natural.

Queremos provar que

$$
F_{2}+F_{4}+F_{6}+\cdots+F_{2 n}+F_{2(n+1)}=F_{2(n+1)+1}-1=F_{2 n+3}-1 .
$$

Somando $F_{2(n+1)}$ à hipótese de indução, temos:

$$
F_{2}+F_{4}+F_{6}+\cdots+F_{2 n}+F_{2(n+1)}=F_{2 n+1}+F_{2(n+1)}-1 .
$$

Observe que $F_{2 n+1}+F_{2(n+1)}=F_{2 n+3}$. Logo,

$$
F_{2}+F_{4}+F_{6}+\cdots+F_{2 n}+F_{2(n+1)}=F_{2(n+1)+1}-1=F_{2 n+3}-1 .
$$

O que prova o resultado para todo $n$ natural.

Proposição 1.8. $F_{1}+F_{3}+F_{5}+\cdots+F_{2 n-1}=F_{2 n}$, para todo $n$ natural.

Demonstração. Observe que $F_{1}=F_{2 \cdot 1}=1$ e que $F_{1}+F_{3}=F_{2 \cdot 2}=F_{4}=3$. Agora suponha que para um certo $n$ natural

$$
F_{1}+F_{3}+F_{5}+\cdots+F_{2 n-1}=F_{2 n} \text { seja verdadeira. }
$$

Queremos provar que

$$
F_{1}+F_{3}+F_{5}+\cdots+F_{2 n-1}+F_{2 n+1}=F_{2 n+2} .
$$

Somando $F_{2 n+1}$ à hipótese de indução, temos:

$$
F_{1}+F_{3}+F_{5}+\cdots+F_{2 n-1}+F_{2 n+1}=F_{2 n}+F_{2 n+1}=F_{2 n+2} .
$$

Portanto, pelo princípio de indução finita, $F_{1}+F_{3}+F_{5}+\cdots+F_{2 n-1}=F_{2 n}$, para todo $n$ natural.

Proposição 1.9. $\left(F_{1}\right)^{2}+\left(F_{2}\right)^{2}+\left(F_{3}\right)^{2}+\cdots+\left(F_{n}\right)^{2}=F_{n} F_{n+1}$, para todo $n$ natural. Demonstração. De fato, $\left(F_{1}\right)^{2}=F_{1} F_{2}=1$ e $\left(F_{1}\right)^{2}+\left(F_{2}\right)^{2}=F_{2} F_{3}=2$, mostrando a base de indução. Suponha que 


$$
\left(F_{1}\right)^{2}+\left(F_{2}\right)^{2}+\left(F_{3}\right)^{2}+\cdots+\left(F_{n}\right)^{2}=F_{n} F_{n+1},
$$

para um certo valor $n$ natural. Queremos provar que

$$
\left(F_{1}\right)^{2}+\left(F_{2}\right)^{2}+\left(F_{3}\right)^{2}+\cdots+\left(F_{n}\right)^{2}+\left(F_{n+1}\right)^{2}=F_{n+1} F_{n+2} .
$$

Adicionando $\left(F_{n+1}\right)^{2}$ à hipótese de indução, temos:

$$
\left(F_{1}\right)^{2}+\left(F_{2}\right)^{2}+\left(F_{3}\right)^{2}+\cdots+\left(F_{n}\right)^{2}+\left(F_{n+1}\right)^{2}=F_{n} F_{n+1}+\left(F_{n+1}\right)^{2} .
$$

Colocando $F_{n+1}$ em evidência, do lado direito da última igualdade, e utilizando a definição recursiva da sequência de Fibonacci, obtemos:

$$
\left(F_{1}\right)^{2}+\left(F_{2}\right)^{2}+\left(F_{3}\right)^{2}+\cdots+\left(F_{n}\right)^{2}+\left(F_{n+1}\right)^{2}=F_{n+1}\left(F_{n}+F_{n+1}\right)=F_{n+1} F_{n+2} .
$$

Assim, pelo princípio de indução, o resultado é verdadeiro para todo $n$ natural.

A Proposição 1.9 mostra um resultado de relevante interpretação geométrica, a saber: a soma das áreas dos primeiros $n$ quadrados cujos lados são os primeiros $n$ números de Fibonacci respectivamente é equivalente à área de um retângulo cujos lados são $F_{n}$ e $F_{n+1}$.

Os quadrados podem ser encaixados para formar o retângulo abaixo. Em cada quadrado foi traçado um quarto de círculo formando a surpreendente espiral de Fibonacci, ${ }^{4}$ ilustrada na figura seguinte, a qual traduz visualmente a ideia da Proposição 1.9.

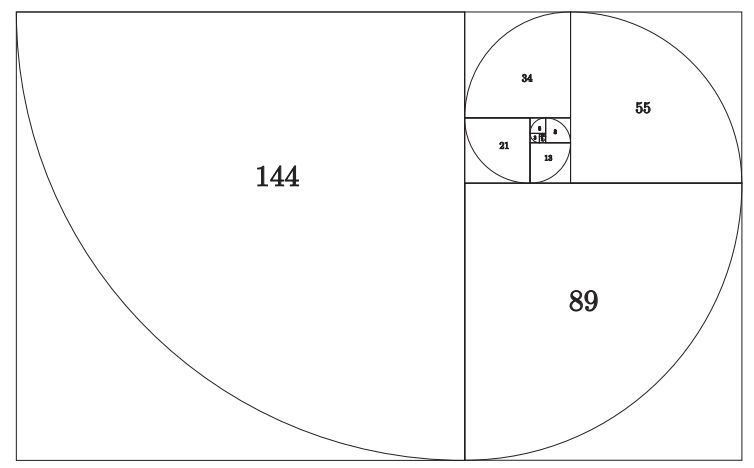

Figura 1.2: Espiral de Fibonacci

Excelentes trabalhos geométricos já foram feitos com esse tema e existem boas fontes de pesquisa conhecidas, as quais podem ser exploradas por aqueles que tenham interesse. Por exemplo, em [5] e [2], várias informações são encontradas.

\footnotetext{
${ }^{4} \mathrm{~A}$ espiral de Fibonacci aproxima-se de uma espiral logarítmica.
} 
Muitos autores abordam a relação entre o retângulo construído na Figura 1.2 e a razão áurea. Mas apesar da vasta literatura sobre razão áurea, não entraremos profundamente nessa seara. Em relação a ela, vamos nos limitar a comentar sobre o limite abaixo.

$$
\lim _{n \rightarrow \infty} \frac{F_{n+1}}{F_{n}}=\frac{1+\sqrt{5}}{2}
$$

\subsection{Fibonacci e algumas relações interessantes}

$\mathrm{Na}$ terceira seção deste capítulo introdutório, vamos abordar outros tópicos matemáticos nos quais é encontrada a sequência de Fibonacci e também demonstrar a ligação dos números de Fibonacci ao número de ouro $(1+\sqrt{5}) / 2 \approx 1,618$.

O primeiro exemplo é sobre o triângulo de Pascal, o qual tem muitas propriedades interessantes conhecidas.

Os números de Fibonacci também são encontrados no triângulo de Pascal quando são somados os números nas diagonais paralelas conforme a Figura 1.3.

Exemplo 1.10 (Fibonacci e triângulo de Pascal). A soma dos números nas diagonais paralelas, conforme a figura abaixo, produz números de Fibonacci.

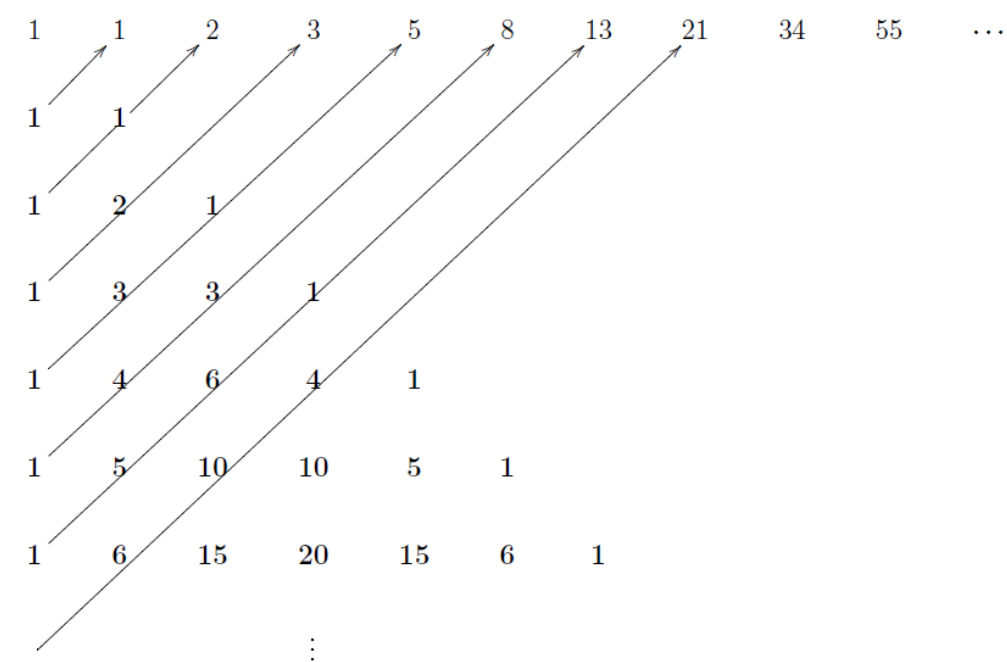

Figura 1.3: Soma das diagonais do triângulo de Pascal

A justificativa desse fato é devida ao matemático francês Édouard Lucas, que, em 1876, escreveu o termo geral da sequência de Fibonacci usando coeficientes binomiais. 
Teorema 1.11. $F_{n+1}=\left(\begin{array}{l}n \\ 0\end{array}\right)+\left(\begin{array}{c}n-1 \\ 1\end{array}\right)+\left(\begin{array}{c}n-2 \\ 2\end{array}\right)+\cdots+\left(\begin{array}{c}n-j \\ j\end{array}\right)$, onde $j$ é o maior inteiro menor do que ou igual a $n / 2$.

Demonstração. Vamos demonstrar utilizando indução sobre $n$. Observe que para $n=$ 0,1 e 2 o resultado é válido. Suponha que para todo inteiro $k, 0 \leq k<n$, a afirmação seja verdadeira. Queremos mostrar que ela vale para $k+1=n$.

Sabemos que $F_{k+1}=F_{k}+F_{k-1}$. Pela hipótese de indução, temos:

$$
\begin{aligned}
F_{k+1}= & F_{k}+F_{k-1} \\
= & {\left[\left(\begin{array}{c}
k-1 \\
0
\end{array}\right)+\left(\begin{array}{c}
k-2 \\
1
\end{array}\right)+\left(\begin{array}{c}
k-3 \\
2
\end{array}\right)+\cdots+\left(\begin{array}{c}
k-j-1 \\
j
\end{array}\right)\right] } \\
& +\left[\left(\begin{array}{c}
k-2 \\
0
\end{array}\right)+\left(\begin{array}{c}
k-3 \\
1
\end{array}\right)+\left(\begin{array}{c}
k-4 \\
2
\end{array}\right)+\cdots+\left(\begin{array}{c}
k-j-1 \\
j-1
\end{array}\right)\right] \\
= & \left(\begin{array}{c}
k-1 \\
0
\end{array}\right)+\left[\left(\begin{array}{c}
k-2 \\
0
\end{array}\right)+\left(\begin{array}{c}
k-2 \\
1
\end{array}\right)\right]+\left[\left(\begin{array}{c}
k-3 \\
1
\end{array}\right)+\left(\begin{array}{c}
k-3 \\
2
\end{array}\right)\right] \\
& +\cdots+\left[\left(\begin{array}{c}
k-j-1 \\
j-1
\end{array}\right)+\left(\begin{array}{c}
k-j-1 \\
j
\end{array}\right)\right] \\
= & \left(\begin{array}{c}
k-1 \\
0
\end{array}\right)+\left(\begin{array}{c}
k-1 \\
1
\end{array}\right)+\left(\begin{array}{c}
k-2 \\
2
\end{array}\right)+\cdots+\left(\begin{array}{c}
k-j \\
j
\end{array}\right) .
\end{aligned}
$$

Isto é, $F_{k+1}=\left(\begin{array}{c}k-1 \\ 0\end{array}\right)+\left(\begin{array}{c}k-1 \\ 1\end{array}\right)+\left(\begin{array}{c}k-2 \\ 2\end{array}\right)+\cdots+\left(\begin{array}{c}k-j \\ j\end{array}\right)$. Onde usamos a conhecida relação de Stifel ${ }^{5}$.

Observando que $\left(\begin{array}{c}k-1 \\ 0\end{array}\right)=\left(\begin{array}{l}k \\ 0\end{array}\right)$, temos $F_{k+1}=\left(\begin{array}{c}k \\ 0\end{array}\right)+\left(\begin{array}{c}k-1 \\ 1\end{array}\right)+\left(\begin{array}{c}k-2 \\ 2\end{array}\right)+\cdots+\left(\begin{array}{c}k-j \\ j\end{array}\right)$.

Portanto, pelo princípio de indução, a afirmação é verdadeira para todo $n$ inteiro não negativo.

O triângulo de Pascal é uma ferramenta riquíssima para explorar conceitos interdisciplinares na Matemática. Além das suas inúmeras propriedades e da relação com o binômio de Newton, o professor pode introduzir os conceitos de sequências numéricas e progressões, trabalhar progressões aritméticas de ordem $n$, achando determinados termos com o auxílio dos sistemas de equações lineares e, além disso, deixar que os alunos explorem esse triângulo no intuito de perceber padrões. Para mais informações sobre o triângulo de Pascal consulte [11].

No Capítulo 3 desse trabalho, encontra-se a Tabela 3.2. Da mesma maneira que é possível explorar padrões numéricos no triângulo de Pascal, é possível utilizar a

$$
5\left(\begin{array}{l}
n \\
p
\end{array}\right)+\left(\begin{array}{c}
n \\
p+1
\end{array}\right)=\left(\begin{array}{c}
n+1 \\
p+1
\end{array}\right) .
$$


referida tabela para trabalhar em sala a habilidade dos alunos em explorar padrões e fazer inferências. No decorrer do texto, serão abordados os conceitos necessários para o professor poder conduzir esse tipo de atividade.

Outro fato interessante sobre os números de Fibonacci é que todo número natural pode ser representado como a soma de números de Fibonacci distintos e não consecutivos. Esse fato é conhecido como Teorema de Zeckendörff.

Para exemplificar, observe que $1,2,3,5$ e 8 são números de Fibonacci e $4=F_{4}+$ $F_{1}, 6=F_{5}+F_{1}, 7=F_{5}+F_{3}, 9=F_{6}+F_{1}, 10=F_{6}+F_{3}$ e $11=F_{6}+F_{4}$ são somas de números de Fibonacci diferentes e não consecutivos.

A prova será feita por indução.

Teorema 1.12 (Teorema de Zeckendörff). Todo inteiro positivo pode ser escrito como soma de números de Fibonacci distintos e não consecutivos.

Demonstração. Observe que a base de indução está bem definida, pois $n=1=F_{1}$. Suponha que o resultado seja verdadeiro para um certo natural $n$, ou seja,

$$
n=F_{m_{1}}+F_{m_{2}}+\cdots+F_{m_{k}}, \operatorname{com} m_{i+1}-m_{i} \geq 2 \text {, para } i \in\{1,2, \ldots, k-1\} .
$$

Queremos mostrar que $n+1$ pode ser escrito como soma de números de Fibonacci distintos e não consecutivos, ou seja,

$$
n+1=1+F_{m_{1}}+F_{m_{2}}+\cdots+F_{m_{k}} .
$$

Note que se $m_{1} \geq 3$, o resultado está provado pois $1=F_{1}$ e portanto

$$
n+1=1+F_{m_{1}}+F_{m_{2}}+\cdots+F_{m_{k}} \text { e } m_{1}-1 \geq 2 .
$$

Sem perda de generalidade, vamos supor que $m_{1}=2$, pois $F_{1}=F_{2}=1$. Assim

$$
n+1=\underbrace{F_{1}+F_{2}}_{F_{3}}+F_{m_{2}}+\cdots+F_{m_{k}}=F_{3}+F_{m_{2}}+\cdots+F_{m_{k}} .
$$

Agora observe que se $m_{2} \geq 5$ o resultado está provado. Supondo $m_{2}=4$, temos:

$$
n+1=\underbrace{F_{3}+F_{4}}_{F_{5}}+F_{m_{3}}+\cdots+F_{m_{k}} \text { e } \log 0 n+1=F_{5}+F_{m_{3}}+\cdots+F_{m_{k}} .
$$

Continuando esse processo, teremos somente um número de Fibonacci ou a soma de números de Fibonacci diferentes e não consecutivos. O que mostra, por indução, o teorema de Zeckendörff. 
Para exemplificar o Teorema 1.12, observe que $2015=F_{17}+F_{14}+F_{9}+F_{5}+F_{3}=$ $1597+377+34+5+2$. Essa representação é única, a menos da ordem das parcelas da soma, quando consideramos números de Fibonacci distintos e não consecutivos.

A seguir vamos relacionar a sequência de Fibonacci à razão áurea e posteriormente, encerrando essa seção, associá-la às triplas pitagóricas.

Para o que segue, a definição algébrica de razão áurea é a seguinte.

Definição 1.13 (Razão Áurea). A razão áurea é uma relação matemática definida algebricamente pela expressão $(a+b) / a=a / b=\alpha$, em que a e $b$ representam números reais, e $\alpha$ representa uma constante de valor aproximadamente igual a 1,618.

A partir da definição algébrica $(a+b) / a=a / b=\alpha$, verifica-se que $1+b / a=\alpha$, isto é, $1+\alpha^{-1}=\alpha$. Multiplicando a última igualdade por $\alpha$, obtemos: $\alpha^{2}-\alpha-1=0$, com raízes $\alpha=(1+\sqrt{5}) / 2$ e $\beta=(1-\sqrt{5}) / 2$.

É possível mostrar que

$$
\lim _{n \rightarrow \infty} \frac{F_{n+1}}{F_{n}}=\frac{1+\sqrt{5}}{2} .
$$

Para mostrarmos esse fato, vamos inicialmente demonstrar, utilizando a fórmula de Binet, as duas identidades seguintes.

Proposição 1.14. $F_{2 n+2} F_{2 n+1}-F_{2 n} F_{2 n+3}=1$.

Demonstração. Utilizando a fórmula de Binet, temos que:

$$
\begin{aligned}
F_{2 n+2} F_{2 n+1}-F_{2 n} F_{2 n+3}= & \frac{1}{5}\left[\left(\alpha^{2 n+2}-\beta^{2 n+2}\right)\left(\alpha^{2 n+1}-\beta^{2 n+1}\right)\right] \\
& -\frac{1}{5}\left[\left(\alpha^{2 n}-\beta^{2 n}\right)\left(\alpha^{2 n+3}-\beta^{2 n+3}\right)\right] \\
= & \frac{1}{5}\left(\alpha^{4 n+3}+\beta^{4 n+3}-\alpha^{2 n+2} \beta^{2 n+1}-\beta^{2 n+2} \alpha^{2 n+1}\right) \\
& -\frac{1}{5}\left(\alpha^{4 n+3}+\beta^{4 n+3}-\alpha^{2 n} \beta^{2 n+3}-\beta^{2 n} \alpha^{2 n+3}\right) \\
= & \frac{1}{5}\left(-\alpha^{2 n+2} \beta^{2 n+1}-\beta^{2 n+2} \alpha^{2 n+1}+\alpha^{2 n} \beta^{2 n+3}+\beta^{2 n} \alpha^{2 n+3}\right) \\
= & \frac{1}{5}\left[-(\alpha \beta)^{2 n+1} \alpha-(\beta \alpha)^{2 n+1} \beta+(\alpha \beta)^{2 n} \beta^{3}+(\beta \alpha)^{2 n} \alpha^{3}\right] \\
= & \frac{1}{5}\left(\alpha+\beta+\beta^{3}+\alpha^{3}\right) \\
= & \frac{1}{5}(1+4)=1,
\end{aligned}
$$

onde usamos que $\alpha \beta=-1$. 
Proposição 1.15. $F_{n}^{2}=F_{n-1} F_{n+1}-(-1)^{n}$.

Demonstração. Utilizando a fórmula de Binet, temos que:

$$
\begin{aligned}
F_{n-1} F_{n+1}-(-1)^{n} & =\frac{\left(\alpha^{n-1}-\beta^{n-1}\right)\left(\alpha^{n+1}-\beta^{n+1}\right)}{(\alpha-\beta)^{2}}-(-1)^{n} \\
& =\frac{\alpha^{2 n}-(\alpha \beta)^{n} \alpha^{-1} \beta-(\alpha \beta)^{n} \beta^{-1} \alpha+\beta^{2 n}}{(\alpha-\beta)^{2}}-(\alpha \beta)^{n} \\
& =\frac{\alpha^{2 n}-(\alpha \beta)^{n}\left(\alpha^{-1} \beta+\beta^{-1} \alpha\right)+\beta^{2 n}}{(\alpha-\beta)^{2}}-(\alpha \beta)^{n} \\
& =\frac{\alpha^{2 n}-(\alpha \beta)^{n}(-\beta \beta-\alpha \alpha)+\beta^{2 n}}{(\alpha-\beta)^{2}}-(\alpha \beta)^{n} \\
& =\frac{\alpha^{2 n}+(\alpha \beta)^{n}\left(\beta^{2}+\alpha^{2}\right)+\beta^{2 n}}{(\alpha-\beta)^{2}}-(\alpha \beta)^{n} \\
& =\frac{\alpha^{2 n}+(\alpha \beta)^{n}(\beta+1+\alpha+1)+\beta^{2 n}}{(\alpha-\beta)^{2}}-(\alpha \beta)^{n} \\
& =\frac{\alpha^{2 n}+3(\alpha \beta)^{n}+\beta^{2 n}}{(\alpha-\beta)^{2}}-(\alpha \beta)^{n} \\
& =\frac{\alpha^{2 n}+3(\alpha \beta)^{n}+\beta^{2 n}-5(\alpha \beta)^{n}}{(\alpha-\beta)^{2}} \\
& =\frac{\alpha^{2 n}-2(\alpha \beta)^{n}+\beta^{2 n}}{(\alpha-\beta)^{2}} \\
& =\frac{\left(\alpha^{n}-\beta^{n}\right)^{2}}{(\alpha-\beta)^{2}} \\
& =F_{n}^{2} . \beta \alpha^{n}-\beta
\end{aligned}
$$

Observe que, nas manipulações algébricas acima, usamos os seguintes fatos conhecidos: $\alpha+\beta=1, \alpha \beta=-1, \alpha^{-1}=-\beta, \beta^{-1}=-\alpha, \alpha^{2}=\alpha+1$ e $\beta^{2}=\beta+1$.

Voltando à análise de

$$
\lim _{n \rightarrow \infty} \frac{F_{n+1}}{F_{n}}=\frac{1+\sqrt{5}}{2}
$$

sejam $r_{n}=F_{n+1} / F_{n}$ a razão entre dois números de Fibonacci consecutivos e $I_{n}=\left[r_{2 n-1}, r_{2 n}\right], n=1,2,3, \ldots$ uma sequência de intervalos fechados, tais que $I_{1} \supseteq I_{2} \supseteq \cdots \supseteq I_{n}$. O teorema dos intervalos encaixantes, ou teorema de Cantor, 
ver [10], afirma que se $I_{1}, I_{2}, I_{3}, \ldots$ é uma sequência de intervalos fechados e limitados, e se o comprimento de $I_{n}$ tende a zero quando $n$ tende ao infinito, então existe um, e somente um, número real que pertence a todos os intervalos da sequência.

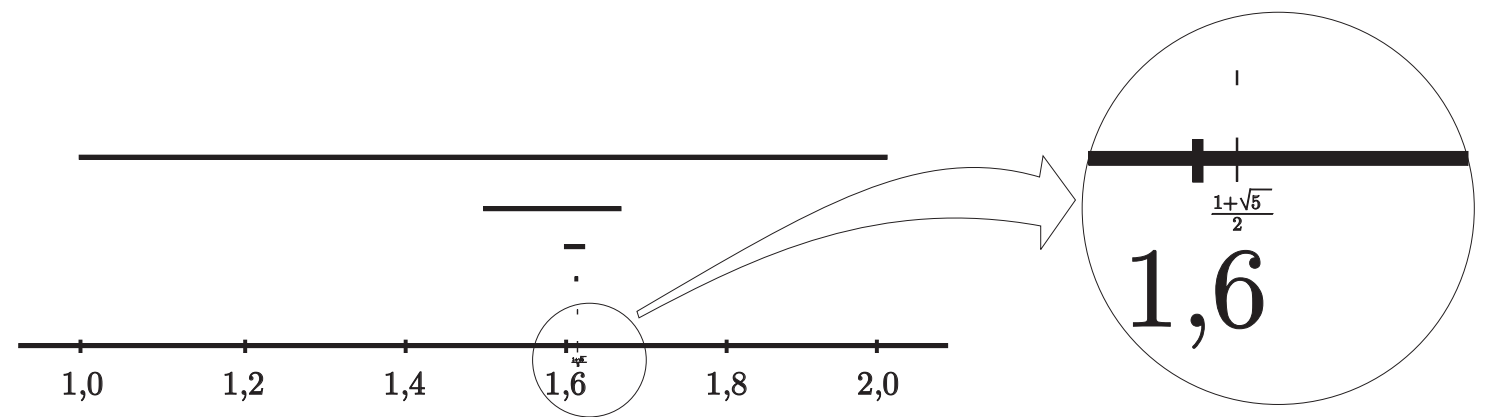

Figura 1.4: Intervalos encaixantes

Para ver que $I_{n}$ é encaixante, observe que a sequência $r_{n}$ possui duas subsequências monótonas, a saber:

$$
r_{2}>r_{4}>\cdots>r_{2 n}>r_{2 n+2}>\cdots \text { e } r_{1}<r_{3}<\cdots<r_{2 n-1}<r_{2 n+1}<\cdots
$$

Mostrar o fato $r_{2 n}>r_{2 n+2}$ é equivalente a demonstrar a desigualdade $F_{2 n+1} / F_{2 n}>F_{2 n+3} / F_{2 n+2}$, ou seja, vamos mostrar que $F_{2 n+2} F_{2 n+1}-F_{2 n} F_{2 n+3}>0$. Para isso, basta dividir a Proposição 1.14 por $F_{2 n+2} F_{2 n}$. Observe.

$\frac{F_{2 n+2} F_{2 n+1}}{F_{2 n+2} F_{2 n}}-\frac{F_{2 n} F_{2 n+3}}{F_{2 n+2} F_{2 n}}=\frac{1}{F_{2 n+2} F_{2 n}} \Leftrightarrow \frac{F_{2 n+1}}{F_{2 n}}-\frac{F_{2 n+3}}{F_{2 n+2}}=\frac{1}{F_{2 n+2} F_{2 n}}>0 \Rightarrow r_{2 n}>r_{2 n+2}$.

Analogamente, é possível mostrar que $r_{2 n-1}<r_{2 n+1}$ para todo $n \in \mathbb{N}$.

Além disso, é possível mostrar que $r_{2 n-1}<r_{2 n}$ para todo $n$ natural. De fato, com auxílio da Proposição 1.15, temos $F_{2 n}^{2}=F_{2 n-1} F_{2 n+1}-(-1)^{2 n}$.

Dividindo a igualdade anterior por $F_{2 n} F_{2 n-1}$, temos:

$$
\frac{F_{2 n+1}}{F_{2 n}}-\frac{F_{2 n}}{F_{2 n-1}}=\frac{1}{F_{2 n-1} F_{2 n+1}} \text {. }
$$

Portanto, a partir da igualdade imediatamente acima, obtemos que:

$$
r_{2 n}-r_{2 n-1}=\frac{1}{F_{2 n-1} F_{2 n+1}}>0 \text { para todo } n \in \mathbb{N} \text { e }
$$




$$
r_{2 n}-r_{2 n-1}=\frac{1}{F_{2 n-1} F_{2 n+1}} \rightarrow 0 \text { quando } n \rightarrow \infty
$$

Uma vez que a sequência dos intervalos fechados $\left[r_{1}, r_{2}\right],\left[r_{3}, r_{4}\right],\left[r_{5}, r_{6}\right], \ldots$ é encaixante e o tamanho de $I_{n}=\left[r_{2 n-1}, r_{2 n}\right]$ tende a zero quando $n$ tende ao infinito, então existe $L \in \mathbb{R}$ tal que

$$
L=\lim _{n \rightarrow \infty} \frac{F_{n+1}}{F_{n}}=\lim _{n \rightarrow \infty} \frac{F_{n}+F_{n-1}}{F_{n}}=\lim _{n \rightarrow \infty}\left(1+\frac{F_{n-1}}{F_{n}}\right)=1+\frac{1}{L} .
$$

Resolvendo a equação $L=1+1 / L$ e observando, pelo contexto do problema, a raiz positiva $L=(1+\sqrt{5}) / 2$, temos que a razão entre dois números de Fibonacci consecutivos tende a $(1+\sqrt{5}) / 2$, isto é,

$$
\lim _{n \rightarrow \infty} \frac{F_{n+1}}{F_{n}}=\frac{1+\sqrt{5}}{2} .
$$

Exemplo 1.16 (Fibonacci e triplas pitagóricas). Quatro números de Fibonacci consecutivos $F_{k}, F_{k+1}, F_{k+2}$ e $F_{k+3}$ estão relacionados a uma tripla pitagórica primitiva se $F_{k+1}$ e $F_{k+2}$ têm paridades distintas, e relacionados a uma tripla pitagórica se $F_{k+1} e$ $F_{k+2}$ têm paridades iguais, isto é, se $F_{k+1} \equiv F_{k+2}(\bmod 2)$.

Solução. Sabemos que as triplas pitagóricas primitivas (ver [18]) são da forma $a=m^{2}+n^{2}, b=2 m n$ e $c=m^{2}-n^{2}, \operatorname{com}(m, n)=1$ e $m+n$ ímpar.

O fato de $m$ e $n$ terem paridades distintas é para garantir que a tripla pitagórica seja primitiva. De fato, como $(m, n)=1$ temos que $\left(m^{2}, m^{2}+n^{2}\right)=1$ e portanto $(a, c)=\left(m^{2}+n^{2}, m^{2}-n^{2}\right)=\left(2 m^{2}, m^{2}+n^{2}\right)=\left(2, m^{2}+n^{2}\right)$, será igual a 1 se, e somente se, $m^{2}+n^{2}$ é ímpar, ou seja, se, e somente se, $m+n$ é ímpar.

Fazendo $F_{k+1}=n$ e $F_{k+2}=m$, temos $a=\left(F_{k+2}\right)^{2}+\left(F_{k+1}\right)^{2}, b=2 F_{k+2} F_{k+1} \mathrm{e}$ $c=\left(F_{k+2}\right)^{2}-\left(F_{k+1}\right)^{2}=F_{k} F_{k+3}$, exemplificando a relação citada acima.

Observe que os quatro números de Fibonacci 1,1,2 e 3 formam a tripla pitagórica primitiva $(3,4,5)$ e os quatro números seguintes $1,2,3$ e 5 formam a tripla primitiva $(5,12,13)$. Já os números de Fibonacci $2,3,5$ e $8, \operatorname{com} 5 \equiv 3(\bmod 2)$, formam a tripla pitagórica $(16,30,34)$.

\subsection{Números de Lucas}

Vamos considerar outra importante sequência numérica recursiva, a qual tem importantes ligações com a sequência de Fibonacci. A seguir, será introduzida a sequência 
dos números de Lucas. Algumas propriedades importantes dela serão abordadas e isso auxiliará no entendimento dos principais teoremas desse trabalho.

Definição 1.17. A sequência dos números de Lucas $\left(L_{n}\right)_{n \geq 0}$ é definida por $L_{n+2}=$ $L_{n+1}+L_{n}$, para $n \geq 0$, onde $L_{0}=2$ e $L_{1}=1$.

Formalmente, existe distinção entre sequência dos números de Lucas e sequência de Lucas. ${ }^{6}$ A sequência dos números de Lucas é um caso particular da sequência de Lucas.

Os 25 primeiros termos da sequência dos números de Lucas são: 2, 1, 3, 4, 7, 11, 18, 29, 47, 76, 123, 199, 322, 521, 843, 1364, 2207, 3571, 5778, 9349, 15127, 24476, 39603, 64079 e 103682.

Observe que o período dos algarismos das unidades da sequência dos números de Lucas é 12, isto é, o algarismo das unidades de $L_{k}$ é igual ao algarismo das unidades de $L_{k+12}$, para $k$ inteiro não negativo. Note também que $5 \nmid L_{n}$ para todo $n$ inteiro não negativo. Vários outros primos não dividem nenhum número de Lucas. Por exemplo, a sequência A053028 em OEIS ${ }^{7}$ - The On Line Encyclopedia of Integer Sequences - é formada por primos que não dividem números de Lucas. Os primeiros primos com essa propriedade são: 5, 13, 17, 37, 53, 61, 73, 89, 97, 109, 113, 137, 149, 157, 173, 193, 197, $233, \ldots$

Vamos determinar uma expressão geral para os números de Lucas similarmente ao que fizemos com a sequência de Fibonacci.

Sabemos que o termo geral da sequência dos números de Lucas é dado por $L_{n}=$ $c_{1} \alpha^{n}+c_{2} \beta^{n}$, com $L_{0}=2, L_{1}=1$ e $c_{1}, c_{2} \in \mathbb{R}$, onde $\alpha=(1+\sqrt{5}) / 2$ e $\beta=(1-\sqrt{5}) / 2$ são raízes da equação característica $r^{2}-r-1=0$ (ver [11]) da recorrência linear de segunda ordem homogênea $L_{n+2}=L_{n+1}+L_{n}$.

Dessa maneira,

$$
\left\{\begin{array} { l } 
{ L _ { 0 } = 2 = c _ { 1 } \alpha ^ { 0 } + c _ { 2 } \beta ^ { 0 } = c _ { 1 } + c _ { 2 } } \\
{ L _ { 1 } = 1 = c _ { 1 } \alpha ^ { 1 } + c _ { 2 } \beta ^ { 1 } = c _ { 1 } \alpha + c _ { 2 } \beta }
\end{array} \Rightarrow \left\{\begin{array}{l}
c_{1}+c_{2}=2 \\
c_{1} \alpha+c_{2} \beta=1
\end{array} .\right.\right.
$$

Assim,

$$
c_{1}=2-c_{2} \Rightarrow\left(2-c_{2}\right) \alpha+c_{2} \beta=1 \Rightarrow c_{2}=1 \text { e } c_{1}=1 .
$$

\footnotetext{
${ }^{6}$ A definição da sequência de Lucas é encontrada em https://en.wikipedia.org/wiki/Lucas_sequence

${ }^{7}$ https://oeis.org
} 
Portanto,

$$
L_{n}=\alpha^{n}+\beta^{n} .
$$

Dada a expressão geral dos números de Lucas é fácil mostrar a proposição seguinte.

Proposição 1.18. Seja $L_{n}$ o n-ésimo número de Lucas, com $L_{n+2}=L_{n+1}+L_{n}$, $L_{0}=2$ e $L_{1}=1$. Mostre que $L_{n} L_{n+1}=L_{2 n+1}+(-1)^{n}$.

Demonstração. Uma vez que $L_{n}=\alpha^{n}+\beta^{n} \operatorname{com} \alpha=(1+\sqrt{5}) / 2$ e $\beta=(1-\sqrt{5}) / 2$, temos $\alpha \beta=-1$ e $\alpha+\beta=1$. Assim,

$$
\begin{aligned}
L_{n} L_{n+1} & =\left(\alpha^{n}+\beta^{n}\right)\left(\alpha^{n+1}+\beta^{n+1}\right) \\
& =\alpha^{2 n+1}+\alpha^{n} \beta^{n+1}+\beta^{n} \alpha^{n+1}+\beta^{2 n+1} \\
& =\alpha^{2 n+1}+\beta^{2 n+1}+(\alpha \beta)^{n}(\beta+\alpha)=L_{2 n+1}+(-1)^{n} .
\end{aligned}
$$

Vamos mostrar a seguir mais um resultado envolvendo as sequências de Fibonacci e dos números de Lucas e suas respectivas expressões gerais (1.1) e (1.2).

Exemplo 1.19. Mostre que é válida a identidade $\left[\left(L_{n}+\sqrt{5} F_{n}\right) / 2\right]^{k}=\left(L_{n k}+\sqrt{5} F_{n k}\right) / 2$, onde $L_{n}$ e $F_{n}$ denotam, respectivamente, o n-ésimo número de Lucas e o n-ésimo número de Fibonacci e $k$ é um inteiro não negativo.

Solução. De (1.2) temos que $L_{n}=\alpha^{n}+\beta^{n}$ e de (1.1) temos $F_{n}=\left(\alpha^{n}-\beta^{n}\right) / \sqrt{5}$, $\operatorname{com} \alpha=(1+\sqrt{5}) / 2$ e $\beta=(1-\sqrt{5}) / 2$. Assim,

$$
\frac{L_{n}+\sqrt{5} F_{n}}{2}=\frac{1}{2}\left(\alpha^{n}+\beta^{n}+\alpha^{n}-\beta^{n}\right)=\alpha^{n} .
$$

Logo,

$$
\left(\frac{L_{n}+\sqrt{5} F_{n}}{2}\right)^{k}=\left(\alpha^{n}\right)^{k}=\frac{2 \alpha^{n k}}{2}=\frac{\alpha^{n k}+\beta^{n k}+\alpha^{n k}-\beta^{n k}}{2}=\frac{L_{n k}+\sqrt{5} F_{n k}}{2} .
$$

Portanto,

$$
\left(\frac{L_{n}+\sqrt{5} F_{n}}{2}\right)^{k}=\frac{L_{n k}+\sqrt{5} F_{n k}}{2}
$$


Observe que a Proposição 1.18 e o Exemplo 1.19 exploram conceitos que são perfeitamente possíveis de serem trabalhados no ensino básico. Dessa forma, o professor pode, além de relacionar a sequência de Fibonacci à razão áurea, explorar propriedades algébricas desses números. Em demais oportunidades desse texto, serão abordados outros exemplos acessíveis aos alunos do Ensino Fundamental e do Ensino Médio.

Além dos assuntos básicos, vamos explorar outros resultados sobre as sequências de Fibonacci e de Lucas, no Capítulo 2 deste trabalho, os quais nos darão suporte para o principal objetivo deste texto, que é o estudo da ordem de aparição na sequência de Fibonacci.

Para encerrar esse primeiro capítulo, vamos caracterizar a soma dos $n$ primeiros números de Lucas e generalizar a ideia para qualquer sequência recorrente do tipo $G_{n+2}=G_{n+1}+G_{n}$, com $G_{1}=a$ e $G_{2}=b, a$ e $b$ reais quaisquer.

Proposição 1.20. $L_{1}+L_{2}+L_{3}+\cdots+L_{n}=L_{n+2}-3$.

Demonstração. Observe que $L_{1}=L_{3}-3$ e $L_{1}+L_{2}=L_{4}-3$. Suponha, por hipótese de indução, que

$$
L_{1}+L_{2}+L_{3}+\cdots+L_{n}=L_{n+2}-3
$$

para algum valor $n$ natural. Queremos mostrar que a relação é válida para $n+1$, isto é, queremos mostrar que

$$
L_{1}+L_{2}+L_{3}+\cdots+L_{n}+L_{n+1}=L_{n+3}-3 .
$$

Somando $L_{n+1}$ a ambos os lados da igualdade, na hipótese de indução, temos a seguinte identidade

$$
L_{1}+L_{2}+L_{3}+\cdots+L_{n}+L_{n+1}=L_{n+1}+L_{n+2}-3 .
$$

Com a definição recorrente da sequência dos números de Lucas, temos:

$$
L_{1}+L_{2}+L_{3}+\cdots+L_{n}+L_{n+1}=L_{n+3}-3 .
$$

Portanto, pelo princípio de indução, a Proposição 1.20 é verdadeira para todo $n$ natural.

Proposição 1.21. Se $G_{n+2}=G_{n+1}+G_{n}, n \geq 1$, com $G_{1}=a$ e $G_{2}=b$, a e b reais quaisquer, então $G_{1}+G_{2}+G_{3}+\cdots+G_{n}=G_{n+2}-G_{2}$.

Demonstração. Observe que $G_{1}=a=(a+b)-b=G_{3}-G_{2}$ e $G_{1}+G_{2}=a+b=$ $(a+2 b)-b=G_{4}-G_{2}$. Suponha que $G_{1}+G_{2}+G_{3}+\cdots+G_{n}=G_{n+2}-G_{2}$ seja válida para um certo $n$ natural. Queremos provar que a afirmação é válida para $n+1$, isto é, 


$$
G_{1}+G_{2}+G_{3}+\cdots+G_{n}+G_{n+1}=G_{n+3}-G_{2} .
$$

Por hipótese de indução, temos:

$$
G_{1}+G_{2}+G_{3}+\cdots+G_{n}=G_{n+2}-G_{2} .
$$

Somando $G_{n+1}$ a ambos os lados da igualdade anterior, temos:

$$
G_{1}+G_{2}+G_{3}+\cdots+G_{n}+G_{n+1}=G_{n+2}+G_{n+1}-G_{2}=G_{n+3}-G_{2} .
$$

Assim, verifica-se, pelo princípio de indução, que a proposição é verdadeira para todo $n$ natural. 


\section{CAPÍTULO 2}

\section{DIVISIBILIDADE E NÚMEROS DE FIBONACCI E DE LUCAS}

\subsection{Resultados clássicos sobre divisibilidade}

Nesse capítulo, vamos demonstrar alguns teoremas sobre as sequências de Fibonacci e dos números de Lucas, os quais abordam ideias e conceitos sobre divisibilidade. Alguns deles nos darão base para as demonstrações sobre os resultados que envolvam a ordem de aparição na sequência de Fibonacci.

Muitos fatos sobre os números de Fibonacci e de Lucas podem ser demonstrados por indução ou usando as conhecidas fórmulas ${ }^{1}$ (1.1) e (1.2). A seguir, demonstraremos alguns deles.

Vamos começar com um exemplo simples, porém importante, utilizando as fórmulas de Binet.

Exemplo 2.1. $F_{2 k}=F_{k} L_{k}$ para todo $k$ inteiro não negativo.

Solução. Utilizando (1.1) e (1.2) temos que

$$
F_{2 k}=\frac{\alpha^{2 k}-\beta^{2 k}}{\alpha-\beta}=\frac{\left(\alpha^{k}\right)^{2}-\left(\beta^{k}\right)^{2}}{\alpha-\beta}=\frac{\left(\alpha^{k}-\beta^{k}\right)\left(\alpha^{k}+\beta^{k}\right)}{\alpha-\beta}=F_{k} L_{k} .
$$

\footnotetext{
${ }^{1}$ Fórmulas de Binet.
} 
A seguir, serão apresentados mais dois exemplos sobre divisibilidade na sequência de Fibonacci. No primeiro exemplo, o objetivo é mostrar que todo número de Fibonacci cujo índice é múltiplo de 3 é par. No segundo exemplo, vamos mostrar uma relação com os números de Fibonacci cujos índices são múltiplos de cinco.

Exemplo 2.2. Prove que $2 \mid F_{3 m}$.

Solução. Vamos provar por indução. Observe que para $m=1$ o resultado é válido, pois $2 \mid F_{3}=2$. Suponha que $2 \mid F_{3 m}$ para um certo $m$ natural. Queremos mostrar que $2 \mid F_{3(m+1)}$. Observe que

$$
F_{3(m+1)}=F_{3 m+3}=F_{3 m+2}+F_{3 m+1}=2 F_{3 m+1}+F_{3 m} .
$$

Como, por hipótese de indução, $2 \mid F_{3 m}$ e além disso $2 \mid 2 F_{3 m+1}$, temos que

$$
2 \mid F_{3(m+1)}=2 F_{3 m+1}+F_{3 m}
$$

Isso prova a validez do resultado.

Exemplo 2.3. Mostre que $5 \mid F_{5 m}$.

Solução. Analogamente ao exemplo anterior, vamos mostrar o resultado por indução. Observe que para $m=1$ o resultado é válido, pois $5 \mid F_{5}=5$. Suponha que $5 \mid F_{5 m}$ para um certo $m$ natural. Queremos mostrar que $5 \mid F_{5(m+1)}$. Observe que

$$
\begin{aligned}
F_{5(m+1)}=F_{5 m+5} & =F_{5 m+4}+F_{5 m+3} \\
& =2 F_{5 m+3}+F_{5 m+2} \\
& =2\left(F_{5 m+2}+F_{5 m+1}\right)+F_{5 m+2} \\
& =3 F_{5 m+2}+2 F_{5 m+1} \\
& =5 F_{5 m+1}+3 F_{5 m} .
\end{aligned}
$$

Agora, basta observar que $5 \mid 5 F_{5 m+1}$ e $5 \mid 3 F_{5 m}$, uma vez que, por hipótese de indução, $5 \mid F_{5 m}$. Assim, $5 \mid 5 F_{5 m+1}+3 F_{5 m}$, o que equivale a dizer $5 \mid F_{5(m+1)}$. Portanto, pelo princípio de indução Matemática, $5 \mid F_{5 m}$ para todo $m$ inteiro não negativo.

Esses dois últimos exemplos nos conduzem a teoremas mais gerais sobre divisibilidade. Vamos agora demonstrar um dos resultados mais clássicos sobre a sequência de Fibonacci, o qual afirma que $\left(F_{m}, F_{n}\right)=F_{(m, n)}$, ou seja, o máximo divisor comum entre dois números de Fibonacci dados (diferentes de $F_{0}$ ) é um número 
de Fibonacci cujo índice é igual ao máximo divisor comum dos índices dos números de Fibonacci fornecidos.

Para isso, precisamos de alguns resultados auxiliares.

Proposição 2.4. $F_{m+n}=F_{m} F_{n+1}+F_{m-1} F_{n}$ para $m$ e $n$ naturais.

Demonstração. Vamos usar indução sobre $n$. Observe que, para $n=1$, temos $F_{m+1}=$ $F_{m} F_{1+1}+F_{m-1} F_{1}=F_{m}+F_{m-1}$. Ou seja, a igualdade vale para $n=1$. Para $n=2$, temos $F_{m+2}=F_{m} F_{3}+F_{m-1} F_{2}=2 F_{m}+F_{m-1}=F_{m+1}+F_{m}$. Logo, a igualdade também é valida para $n=2$. Queremos mostrar que $F_{m+n+1}=F_{m} F_{n+2}+F_{m-1} F_{n+1}$.

Supondo o resultado válido para todos os índices menores ou iguais a um certo valor $n$, temos que:

$$
\begin{aligned}
F_{m+(n-1)} & =F_{m} F_{n}+F_{m-1} F_{n-1} . \\
F_{m+n} & =F_{m} F_{n+1}+F_{m-1} F_{n} .
\end{aligned}
$$

Somando membro a membro as igualdades (2.1) e (2.2), temos:

$$
F_{m+(n-1)}+F_{m+n}=F_{m} F_{n}+F_{m} F_{n+1}+F_{m-1} F_{n-1}+F_{m-1} F_{n} .
$$

Utilizando a definição recursiva dos números de Fibonacci no lado esquerdo da última igualdade e colocando $F_{m}$ e $F_{m-1}$ em evidência no lado direito, obtemos:

$$
F_{m+n+1}=F_{m}\left(F_{n}+F_{n+1}\right)+F_{m-1}\left(F_{n-1}+F_{n}\right)=F_{m} F_{n+2}+F_{m-1} F_{n+1} .
$$

Portanto, pelo princípio de indução Matemática, $F_{m+n}=F_{m} F_{n+1}+F_{m-1} F_{n}$ para quaisquer $m$ e $n$ naturais.

Ampliando a definição recursiva da sequência de Fibonacci para o conjunto dos inteiros, ou seja, considerando $\left(F_{n}\right)_{n \in \mathbb{Z}}=\{\ldots,-21,13,-8,5,-3,2,-1,1,0,1,1,2,3,5, \ldots\}$, temos que $F_{-n}=(-1)^{n+1} F_{n}$. Com isso e com a Proposição 2.4, podemos mostrar a identidade de d'Ocagne.

Corolário 2.5 (Identidade de d'Ocagne). $(-1)^{n} F_{m-n}=F_{m} F_{n+1}-F_{n} F_{m+1}$.

Demonstração. Vimos que $F_{m+n}=F_{m+1} F_{n}+F_{m} F_{n-1}$. Usando $-n$ no lugar de $n$ na identidade anterior, temos $F_{m-n}=F_{m+1} F_{-n}+F_{m} F_{-n-1}$. Agora, usando a relação 
$F_{-n}=(-1)^{n+1} F_{n}$, temos que $F_{-(n+1)}=F_{-n-1}=(-1)^{n} F_{n+1}$. Substituindo em $F_{m-n}=$ $F_{m+1} F_{-n}+F_{m} F_{-n-1}$ os valores de $F_{-n}$ e $F_{-n-1}$, obtemos:

$$
\begin{aligned}
F_{m-n} & =F_{m+1} F_{-n}+F_{m} F_{-n-1} \\
& =F_{m+1}(-1)^{n+1} F_{n}+F_{m}(-1)^{n} F_{n+1} .
\end{aligned}
$$

Multiplicando a última igualdade por $(-1)^{n}$, temos $(-1)^{n} F_{m-n}=F_{m} F_{n+1}-F_{n} F_{m+1}$. O que mostra o resultado.

A próxima proposição também nos auxiliará na demonstração do Teorema 2.7.

Proposição 2.6. $F_{n} \mid F_{m n}$, para todos $m, n \in \mathbb{N}$.

Demonstração. Vamos demonstrar a Proposição 2.6 utilizando indução em $m$. Para $m=1$ o resultado é trivialmente verificado. Supondo que $F_{n} \mid F_{m n}$ para $m=$ $1,2,3, \ldots, k$, vamos mostrar que $F_{n} \mid F_{(k+1) n}$. Com efeito, pela Proposição 2.4,

$$
F_{(k+1) n}=F_{(k n)+n}=F_{k n} F_{n+1}+F_{k n-1} F_{n} .
$$

Pela hipótese de indução, $F_{n} \mid F_{k n}$ e $F_{n} \mid F_{n}$. Assim $F_{n}$ divide qualquer combinação linear entre eles, ou seja,

$$
F_{n} \mid F_{k n} F_{n+1}+F_{k n-1} F_{n}=F_{(k+1) n} .
$$

Portanto, pelo princípio de indução, $F_{n} \mid F_{m n}$ para todos $m$ e $n$ naturais.

Teorema 2.7. Se $m$ e $n$ são naturais, então $\left(F_{m}, F_{n}\right)=F_{(m, n)}$.

Demonstração. Seja $c=(m, n)$. Assim, $c \mid m$ e $c \mid n$ e, pela Proposição 2.6, $F_{c} \mid F_{m}$ e também $F_{c} \mid F_{n}$. Portanto, $F_{c}$ é um divisor comum de $F_{m}$ e $F_{n}$, de onde segue que $F_{c} \mid d=\left(F_{m}, F_{n}\right)$.

Uma vez que $c=(m, n)$, existem inteiros $a$ e $b$ tais que $c=a m+b n$, ver [7].

Suponha $a \leq 0$ e seja $k=-a$. Dessa forma, $b n=c+k m$ e, pela Proposição 2.4,

$$
F_{b n}=F_{c+k m}=F_{c} F_{k m+1}+F_{c-1} F_{k m} .
$$

Por outro lado, $d \mid F_{m}$ e $d \mid F_{n}$, e pela Proposição 2.6, $F_{m} \mid F_{k m}$ bem como $F_{n} \mid F_{b n}$.

Logo, $d \mid F_{k m}$ e $d \mid F_{b n}$, e assim divide qualquer combinação linear entre eles, em especial, $d \mid F_{b n}-F_{c-1} F_{k m}$, ou seja, $d \mid F_{c} F_{k m+1}$. 
Sabemos, pela Proposição 1.5 , que $\left(F_{k m}, F_{k m+1}\right)=1$ e vimos que $d \mid F_{k m}$, então $\left(d, F_{k m+1}\right)=1$. Sendo assim, $d \mid F_{c}$. Por outro lado, vimos acima que, $F_{c} \mid d$. Como são ambos positivos, $F_{c}$ e $d$, temos que $\left(F_{m}, F_{n}\right)=d=F_{c}=F_{(m, n)}$ e a demonstração está completa.

Corolário 2.8. $m\left|n \Longleftrightarrow F_{m}\right| F_{n}$, para $m \geq 3$.

Demonstração. Segue do Teorema 2.7 que

$$
m\left|n \Longleftrightarrow(m, n)=m \Longleftrightarrow F_{(m, n)}=F_{m} \Longleftrightarrow\left(F_{m}, F_{n}\right)=F_{m} \Longleftrightarrow F_{m}\right| F_{n} .
$$

Como aplicação do teorema e do corolário anterior, segue mais um exemplo sobre divisibilidade.

Exemplo 2.9. Mostre que $2\left|F_{m} \Longleftrightarrow 3\right|$ m e $3\left|F_{m} \Longleftrightarrow 4\right| m$.

Solução. Observe que $2 \mid F_{m} \Longleftrightarrow F_{(3, m)}=\left(F_{3}, F_{m}\right)=\left(2, F_{m}\right)=2=F_{3}$. Portanto, $2 \mid F_{m} \Longleftrightarrow(3, m)=3$, isto é, se e somente se $3 \mid m$.

Analogamente, $3 \mid F_{m} \Longleftrightarrow F_{(4, m)}=\left(F_{4}, F_{m}\right)=\left(3, F_{m}\right)=3=F_{4}$. Portanto, $3 \mid F_{m} \Longleftrightarrow(4, m)=4$, ou seja, se e somente se $4 \mid m$.

Exemplo 2.10. $2 \mid L_{m}$ se, e somente se, $3 \mid \mathrm{m}$.

Solução. Sabendo que a soma de dois números é par se, e somente se, os números têm paridades iguais, basta observar os primeiros termos da sequência dos números de Lucas. $L_{0}=2, L_{1}=1, L_{2}=3, L_{3}=4, L_{4}=7, L_{5}=11, L_{6}=18, \ldots$ onde observa-se o seguinte padrão de repetição: par, ímpar, ímpar. O qual se repete infinitamente, ou seja, a cada três números consecutivos de Lucas um é par. Portanto, se o índice $m$ do número de Lucas é múltiplo de 3 então $L_{m}$ é par. Equivalentemente $2 \mid L_{m}$ se, e somente se, $3 \mid \mathrm{m}$.

No primeiro capítulo, afirmamos que a periodicidade dos algarismos das unidades dos números de Fibonacci tem tamanho 60. Agora vamos mostrar esse fato.

Proposição 2.11. $F_{k+60} \equiv F_{k}(\bmod 10)$ para todo $k$ inteiro não negativo.

Demonstração. Observe que $F_{60} \equiv F_{0}(\bmod 10)$, pois $10 \mid F_{60}=1.548 .008 .755 .920 . \mathrm{E}$ também $F_{61} \equiv F_{1}(\bmod 10)$, pois $10 \mid F_{61}-F_{1}=2.504 .730 .781 .961-1$. Suponha que $F_{k+60} \equiv F_{k}(\bmod 10)$ para um certo $k$ natural. Queremos provar que $F_{k+1+60} \equiv F_{k+1}$ 
(mod 10). Por hipótese de indução, temos que $F_{k+60} \equiv F_{k}(\bmod 10)$. Somando $F_{k+59}$ à congruência anterior, temos $F_{k+59}+F_{k+60} \equiv F_{k}+F_{k+59}(\bmod 10)$. Usando a Proposição 2.4 em $F_{k+59}$, obtemos

$$
F_{k+61} \equiv F_{k}+F_{k} F_{60}+F_{k-1} F_{59} \equiv F_{k}+F_{k-1}=F_{k+1}(\bmod 10),
$$

uma vez que $F_{60} \equiv 0(\bmod 10)$ e $F_{59} \equiv 1(\bmod 10)$. Portanto, pelo princípio de indução, $F_{k+60} \equiv F_{k}(\bmod 10)$ para todo $k$ inteiro não negativo.

Para que a prova fique completa, precisamos mostrar que 60 é o menor período para o qual os dígitos das unidades, nos números da sequência de Fibonacci, se repetem. De fato, se houvesse um período menor do que 60 ele seria um divisor do próprio 60, ou seja, teria o tamanho de algum dos números do conjunto $\{1,2,3,4,5,6,10,12,15,20,30\}$.

Por inspeção, é fácil perceber que a periodicidade dos dígitos das unidades na sequência de Fibonacci é diferente de qualquer um dos elementos do conjunto dos divisores de 60 menores do que 20 , isto é, diferente de $\{1,2,3,4,5,6,10,12,15\}$ e, tendo em vista que $6765=F_{20} \not \equiv 0(\bmod 10)$ e $1346269=F_{31} \not \equiv F_{1}(\bmod 10)$, o resultado fica completamente provado.

A seguir, vamos demonstrar, utilizando as fórmulas (1.1) e (1.2), uma proposição que relaciona números de Fibonacci com números de Lucas. Ela será importante na construção de fórmulas fechadas para $F_{m} \pm 1$.

Proposição 2.12. Para quaisquer inteiros a e b, temos $F_{a} L_{b}=F_{a+b}+(-1)^{b} F_{a-b}$.

Demonstração. Sabemos que $\alpha=(1+\sqrt{5}) / 2=(-\beta)^{-1}=2 /(\sqrt{5}-1)$ e que $\beta=(-\alpha)^{-1}$. Dessa forma, utilizando (1.1) e (1.2), temos:

$$
\begin{aligned}
F_{a} L_{b} & =\frac{\alpha^{a}-\beta^{a}}{\alpha-\beta}\left(\alpha^{b}+\beta^{b}\right) \\
& =\frac{\alpha^{a+b}-\beta^{a+b}+\alpha^{a} \beta^{b}-\beta^{a} \alpha^{b}}{\alpha-\beta} \\
& =F_{a+b}+\frac{\alpha^{a} \beta^{b}-\beta^{a} \alpha^{b}}{\alpha-\beta} \\
& =F_{a+b}+\frac{\alpha^{a}(-\alpha)^{-b}-\beta^{a}(-\beta)^{-b}}{\alpha-\beta} \\
& =F_{a+b}+(-1)^{b} \cdot \frac{\alpha^{a-b}-\beta^{a-b}}{\alpha-\beta}=F_{a+b}+(-1)^{b} F_{a-b} .
\end{aligned}
$$


A Proposição 2.12 terá grande importância no Capítulo 3. A usaremos para fatorar $F_{m} \pm 1$, dependendo da classe de restos de $m$ módulo 4 . Com ela em mãos, podemos mostrar, de maneira simples, mais um resultado sobre divisibilidade.

O teorema a seguir afirma que, para $n$ par, $F_{n}$ divide a soma de $2 n$ números consecutivos da sequência de Fibonacci.

Teorema 2.13. A soma de $2 n$ números consecutivos da sequência de Fibonacci é divisivel por $F_{n}, \forall n$ par.

Demonstração. Seja $T=F_{a+1}+F_{a+2}+\cdots+F_{a+2 n}$ a soma de $2 n$ números de Fibonacci consecutivos e seja $S_{n}$ a soma dos primeiros $n$ números da sequência de Fibonacci. Assim, $T=S_{a+2 n}-S_{a}$. Sabemos, pela Proposição 1.6, que

$$
S_{n}=F_{1}+F_{2}+F_{3}+\cdots+F_{n}=F_{n+2}-1 .
$$

Logo, $T=F_{a+2 n+2}-F_{a+2}$. Fazendo $q=a+n+2, p=n$ e utilizando a Proposição 2.12, $\operatorname{com} p-q \equiv q(\bmod 2)$, isto é, $p$ par, temos $T=F_{q+p}-F_{q-p}=L_{q} F_{p}=L_{a+n+2} F_{n}$, ou seja, $F_{n}$ divide $T$. O que mostra o resultado.

\subsection{Símbolo de Legendre e resultados usando con- gruências módulo $p$ primo.}

Vamos agora mostrar três resultados importantes usando congruências para $F_{p}, L_{p}$ e $F_{p \pm 1}$ módulo $p$ primo. Esses resultados serão essenciais para o próximo capítulo. Para isso, precisamos relembrar a lei da reciprocidade quadrática de Gauss e o símbolo de Legendre. Maiores informações sobre esses dois últimos tópicos podem ser vistas em [18] e [21].

Definição 2.14. O símbolo de Legendre de a sobre p, denotado por $\left(\frac{a}{p}\right)$, sendo p primo e a um inteiro não múltiplo de p, é tal que:

$$
\left(\frac{a}{p}\right)=\left\{\begin{aligned}
1, & \text { se } x^{2} \equiv a(\bmod p) \text { tem solução } \\
-1, & \text { se } x^{2} \equiv a(\bmod p) \text { não tem soluçã } o
\end{aligned}\right.
$$


A lei da reciprocidade quadrática é uma poderosa ferramenta para calcular o valor do símbolo de Legendre e para calcular se um inteiro $n$ é quadrado módulo $p$ primo.

Abaixo, vamos enunciar a referida lei sem demonstrá-la. Em [19], encontram-se três demonstrações, as quais usam respectivamente os conceitos de combinatória, trigonometria e corpos finitos.

Teorema 2.15 (Lei da reciprocidade quadrática). Sejam p e q primos ímpares distintos. Então

$$
\left(\frac{p}{q}\right)\left(\frac{q}{p}\right)=(-1)^{\frac{p-1}{2} \cdot \frac{q-1}{2}}
$$

A seguir, vamos apresentar um lema que utiliza a lei da reciprocidade quadrática e utilizaremos esse mesmo lema na demonstração dos Teoremas 2.17 e 2.19.

Lema 2.16. Sejam $\left(\frac{5}{p}\right)$ o símbolo de Legendre e $p$ um primo ímpar, $p \neq 2$ e 5 , então

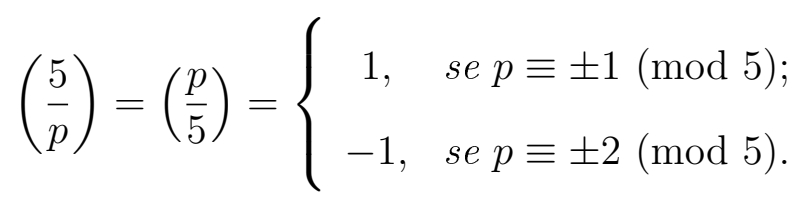

Demonstração. Pelo Teorema 2.15, $\left(\frac{5}{p}\right)=(-1)^{\frac{5-1}{2} \cdot \frac{p-1}{2}}\left(\frac{p}{5}\right)$, ou seja, $\left(\frac{5}{p}\right)=(-1)^{p-1}\left(\frac{p}{5}\right)$. Uma vez que $p$ é primo ímpar, $\left(\frac{5}{p}\right)=\left(\frac{p}{5}\right)$.

Agora, observe que

$$
\left\{\begin{aligned}
p & \equiv 1(\bmod 5) \Rightarrow\left(\frac{p}{5}\right)=\left(\frac{1}{5}\right)=1 \\
p & \equiv 2(\bmod 5) \Rightarrow\left(\frac{p}{5}\right)=\left(\frac{2}{5}\right)=-1 \\
p & \equiv 3(\bmod 5) \Rightarrow\left(\frac{p}{5}\right)=\left(\frac{3}{5}\right)=-1 \\
p & \equiv 4(\bmod 5) \Rightarrow\left(\frac{p}{5}\right)=\left(\frac{4}{5}\right)=1
\end{aligned}\right.
$$

Portanto,

$$
\left(\frac{5}{p}\right)=\left(\frac{p}{5}\right)=\left\{\begin{aligned}
1, & \text { se } p \equiv \pm 1(\bmod 5) \\
-1, & \text { se } p \equiv \pm 2(\bmod 5)
\end{aligned}\right.
$$

Com isso, podemos demonstrar o seguinte teorema que caracteriza $F_{p}$ módulo $p$ primo. 
Teorema 2.17. Seja p um primo impar. Então $F_{p} \equiv\left(\frac{p}{5}\right)(\bmod p)$.

Demonstração. Observe que $\left(\begin{array}{l}p \\ k\end{array}\right) k !=p(p-1)(p-2)(p-3) \cdots(p-(k-1)) \equiv 0(\bmod p)$ e que $p \mid\left(\begin{array}{l}p \\ k\end{array}\right)$ para $0<k<p$. Disto e de (1.1), temos:

$$
\begin{aligned}
F_{p}=\frac{1}{\sqrt{5}}\left(\alpha^{p}-\beta^{p}\right) & =\frac{1}{\sqrt{5} \cdot 2^{p}}\left((1+\sqrt{5})^{p}-(1-\sqrt{5})^{p}\right) \\
& =\frac{1}{\sqrt{5} \cdot 2^{p}}\left(\sum_{k=0}^{p}\left(\begin{array}{l}
p \\
k
\end{array}\right)(\sqrt{5})^{k}-\sum_{k=0}^{p}\left(\begin{array}{l}
p \\
k
\end{array}\right)(-\sqrt{5})^{k}\right) \\
& =\frac{1}{\sqrt{5} \cdot 2^{p}} \sum_{k=0}^{p}\left(\begin{array}{l}
p \\
k
\end{array}\right)\left((\sqrt{5})^{k}-(-\sqrt{5})^{k}\right) \\
& =\frac{1}{2^{p-1}} \sum_{k=0}^{p}\left(\begin{array}{l}
p \\
k \nmid k
\end{array}\right) 5^{\frac{k-1}{2}} .
\end{aligned}
$$

Mas, $\frac{1}{2^{p-1}} \sum_{\substack{k=0 \\ 2 \nmid k}}^{p}\left(\begin{array}{l}p \\ k\end{array}\right) 5^{\frac{k-1}{2}} \equiv 5^{\frac{p-1}{2}}(\bmod p)$. E, pelo critério de Euler (ver [21])

$$
5^{\frac{p-1}{2}} \equiv\left(\frac{5}{p}\right) \equiv\left(\frac{p}{5}\right)(\bmod p) .
$$

Portanto, $F_{p} \equiv\left(\frac{p}{5}\right)(\bmod p)$. O que conclui a demonstração.

Teorema 2.18. Se p é um primo ímpar, então $L_{p} \equiv 1(\bmod p)$.

Demonstração. Similarmente à prova do teorema anterior, considere (1.2), e lembre-se que $\left(\begin{array}{l}p \\ k\end{array}\right) k !=p(p-1)(p-2)(p-3) \cdots(p-(k-1)) \equiv 0(\bmod p)$ e que $p \mid\left(\begin{array}{l}p \\ k\end{array}\right)$ para $0<k<p$.

Dessa forma, $L_{p}=\alpha^{p}+\beta^{p}=\frac{1}{2^{p}} \sum_{k=0}^{p}\left(\begin{array}{l}p \\ k\end{array}\right)\left((\sqrt{5})^{k}+(-\sqrt{5})^{k}\right)=\frac{1}{2^{p-1}} \sum_{\substack{k=0 \\ 2 \mid k}}^{p}\left(\begin{array}{l}p \\ k\end{array}\right) 5^{\frac{k}{2}} \equiv \frac{1}{2^{p-1}} \equiv 1(\bmod p)$.

Portanto, $L_{p} \equiv 1(\bmod p)$.

Teorema 2.19. Temos que $F_{p-1} \equiv \frac{1-\left(\frac{p}{5}\right)}{2}(\bmod p)$ e $F_{p+1} \equiv \frac{1+\left(\frac{p}{5}\right)}{2}(\bmod p)$, para $p$ primo impar. 
Demonstração. Sabemos que $L_{n}=F_{n+1}+F_{n-1}$ para todo $n$ natural. $^{2}$

Logo,

$$
L_{p}=F_{p+1}+F_{p-1}
$$

para todo $p$ primo. Dessa forma, podemos escrever $L_{p}$ das duas maneiras seguintes:

$$
L_{p}=F_{p}+2 F_{p-1} \text { e } L_{p}=2 F_{p+1}-F_{p} \text {. Assim, } F_{p-1}=\left(\frac{L_{p}-F_{p}}{2}\right) \text { e } F_{p+1}=\left(\frac{L_{p}+F_{p}}{2}\right) .
$$

Sabemos, pelos Teoremas 2.17 e 2.18 , respectivamente, que $F_{p} \equiv\left(\frac{p}{5}\right)(\bmod p)$ e $L_{p} \equiv 1(\bmod p) . \quad$ Portanto, substituindo $F_{p} \equiv\left(\frac{p}{5}\right)(\bmod p)$ e $L_{p} \equiv 1(\bmod p)$ nas igualdades anteriores, a demonstração está completa, ou seja, $F_{p-1} \equiv \frac{1-\left(\frac{p}{5}\right)}{2}(\bmod p)$ e $F_{p+1} \equiv \frac{1+\left(\frac{p}{5}\right)}{2}(\bmod p)$.

Corolário 2.20. Seja p um número primo. Então $p \mid F_{p-\left(\frac{p}{5}\right)}$.

Demonstração. Basta observar que: Se $p=5$, então $p \mid F_{p}$, pois $5 \mid F_{5}$. Se $\left(\frac{p}{5}\right)=-1$, então $F_{p+1} \equiv 0(\bmod p)$, ou seja $p \mid F_{p+1}$. E, por último, se $\left(\frac{p}{5}\right)=1$, então $F_{p-1} \equiv$ $0(\bmod p)$, ou seja $p \mid F_{p-1}$. Dessa forma, sendo $p$ um número primo, $p \mid F_{p-\left(\frac{p}{5}\right)}$.

\subsection{Teoremas auxiliares}

Para encerrar esse capítulo, vamos apresentar alguns teoremas envolvendo os números de Fibonacci, os números de Lucas e alguns conceitos vistos até agora. Além disso, vamos fazer um breve comentário sobre somas de $s$-ésimas potências de números de Fibonacci consecutivos.

Teorema 2.21. $L_{n}=F_{n+1}+F_{n-1}$ para todo $n$ natural.

Demonstração. Vamos demonstrar por indução. Observe que para $n=1$ e $n=2$ temos: $L_{1}=F_{2}+F_{0}$ e $L_{2}=F_{3}+F_{1}$.

Suponha que $L_{k}=F_{k+1}+F_{k-1}$ para todo $1 \leq k \leq n$. Queremos mostrar que

$$
L_{n+1}=F_{n+2}+F_{n} .
$$

${ }^{2}$ Essa afirmação é facilmente provada por indução ou utilizando as fórmulas de Binet. Na Seção 2.3, apresentaremos a demonstração por indução. 
Observe que, por hipótese de indução, são válidas as igualdades seguintes.

$$
\begin{aligned}
L_{n-1} & =F_{n}+F_{n-2} . \\
L_{n} & =F_{n+1}+F_{n-1} .
\end{aligned}
$$

Somando (2.3) e (2.4), obtemos $L_{n+1}=F_{n+2}+F_{n}$. O que mostra a validez do resultado para todo $n$ natural.

Analogamente à Proposição 2.4, vamos demonstrar um resultado que envolve números de Lucas cujos índices são dados por uma soma.

Teorema 2.22. $L_{m+n}=F_{m+1} L_{n}+F_{m} L_{n-1}$ para $m$ e $n$ naturais.

Demonstração. Vamos usar indução sobre $n$. Observe que para $n=1$, temos

$$
L_{m+1}=F_{m+1} L_{1}+F_{m} L_{0}=F_{m+1}+2 F_{m} .
$$

Ou seja, $L_{m+1}=F_{m+2}+F_{m}$. Dessa forma a igualdade vale para $n=1$, pois vimos no Teorema 2.21 que $L_{n}=F_{n+1}+F_{n-1}$ para todo $n$.

Para $n=2$, temos $L_{m+2}=3 F_{m+1}+F_{m}=F_{m+3}+F_{m+1}$. Logo, pelo Teorema 2.21, a igualdade também é valida para $n=2$.

Queremos mostrar que $L_{m+(n+1)}=F_{m+1} L_{n+1}+F_{m} L_{n}$.

Supondo o resultado válido para todos os índices menores ou iguais a um certo valor $n$, temos que:

$$
\begin{aligned}
L_{m+(n-1)} & =F_{m+1} L_{n-1}+F_{m} L_{n-2} \\
L_{m+n} & =F_{m+1} L_{n}+F_{m} L_{n-1}
\end{aligned}
$$

Somando membro a membro as igualdades (2.5) e (2.6), temos:

$$
L_{m+(n-1)}+L_{m+n}=F_{m+1} L_{n-1}+F_{m+1} L_{n}+F_{m} L_{n-2}+F_{m} L_{n-1} .
$$

Utilizando a definição recursiva dos números de Lucas na última igualdade e colocando $F_{m+1}$ e $F_{m}$ em evidência, obtemos:

$$
L_{m+n+1}=F_{m+1} L_{n+1}+F_{m} L_{n} .
$$

Portanto, pelo princípio de indução Matemática, $L_{m+n}=F_{m+1} L_{n}+F_{m} L_{n-1}$ para quaisquer $m$ e $n$ naturais. 
Em [3], existe uma generalização do teorema anterior. A ideia é que, dada uma sequência recorrente definida por $G_{1}=p, G_{2}=q$, com $G_{n+2}=G_{n+1}+G_{n}$, a identidade seguinte se verifica.

$$
G_{m+n}=F_{m+1} G_{n}+F_{m} G_{n-1}
$$

O próximo teorema contém alguns resultados que serão úteis na caracterização de $z\left(F_{m} \pm 1\right)$.

Teorema 2.23. Temos que:

a) $L_{n} \mid F_{m}$ se, e somente se, $n$ divide $m$ e $m / n$ é par;

b) $L_{n} \mid L_{m}$ se, e somente se, $n$ divide $m$ e $m / n$ é ímpar;

c) Sed $\operatorname{sdc}(m, n)=(m, n)$, então $\left(F_{m}, L_{n}\right)= \begin{cases}L_{d}, & \text { se } \frac{m}{d} \text { é par e } \frac{n}{d} \text { é ímpar; } \\ 1 \text { ou } 2, & \text { caso contrário. }\end{cases}$

d) $F_{3 n}=5 F_{n}^{3}+3(-1)^{n} F_{n}$;

e) $3 F_{4 n} \mid F_{12 n}$.

Demonstração. De fato, para demonstrar a), basta mostrar que $L_{n} \mid F_{m=2 n k}$ e que $L_{n} \nmid F_{2 n-j}$, para $j=1,2,3, \ldots, 2 n-1$.

Pelo Exemplo 2.1, sabemos que $F_{2 n}=F_{n} L_{n}$ de onde segue que $L_{n} \mid F_{2 n}$. A Proposição 2.6 garante que $F_{2 n} \mid F_{2 n k}$ Portanto, $L_{n} \mid F_{2 n k}$.

Agora, vamos usar a Proposição 2.12 para escrever $F_{2 n-j}=L_{n} F_{n-j}+(-1)^{n} F_{-j}$. Queremos mostrar que $L_{n} \nmid F_{2 n-j}$. Se $L_{n}$ dividisse $F_{2 n-j}$, então dividiria $F_{-j}$. Mas isto é claramente um absurdo, pois $L_{n}>F_{n}>\left|F_{-j}\right|$. Portanto $L_{n} \nmid F_{2 n-j}$. Do exposto, segue que $L_{n} \mid F_{m}$ se, e somente se, $n \mid m$ e $m / n$ é par.

Demonstração de $b)$ :

Queremos mostrar que $L_{n} \mid L_{m}$ se, e somente se, $m=(2 k+1) n$.

Pelo Teorema 2.22, fazendo $m=2 k n$, temos:

$$
L_{2 k n+n}=L_{(2 k+1) n}=F_{2 k n+1} L_{n}+F_{2 k n} L_{n-1} .
$$


Assim, para mostrar que $L_{n}$ divide $L_{(2 k+1) n}$ basta mostrar que $L_{n}$ divide $F_{2 k n}$.

Sabemos, pelo Exemplo 2.1, que $F_{2 n}=F_{n} L_{n}$. Isso implica $L_{n} \mid F_{2 n}$. Por outro lado, pela Proposição 2.6, $F_{2 n} \mid F_{2 n k}$. Portanto $L_{n} \mid F_{2 n k}$. De onde segue que $L_{n} \mid L_{m}$ com $m=(2 k+1) n$.

Tendo em vista que mostramos em a) que $L_{n} \mid F_{m}$ se, e somente se, $m=2 k n$, o resultado fica completamente provado.

\section{Demonstração de c):}

Para demonstrar $c$ ), vamos usar a Proposição 3.7. Observe que, por hipótese, $m / d$ é par e $n / d$ é ímpar. Assim, por a) e por b), respectivamente, $L_{d} \mid F_{m}$ e $L_{d} \mid L_{n}$. Agora, vamos mostrar que $L_{d}=\left(F_{m}, L_{n}\right)$.

De fato, seja $c$ um divisor comum de $F_{m}$ e $L_{n}$. Assim, basta provar que $c \mid L_{d}$.

Observe que $c\left|F_{m} \Rightarrow z(c)\right| m$ (pela Proposição 3.7) e, também, $c\left|L_{n} \Rightarrow z(c)\right| n$.

Como, por hipótese, $(m, n)=d$, temos que $z(c)|d \Rightarrow c| L_{d}$.

As demonstrações dos casos $\left(F_{m}, L_{n}\right)=2$ e $\left(F_{m}, L_{n}\right)=1$ podem ser vistas em [8].

Demonstração de $d)$ :

$$
\begin{aligned}
5 F_{n}^{3}+3(-1)^{n} F_{n} & =\frac{1}{\sqrt{5}}\left[\left(\alpha^{n}-\beta^{n}\right)^{3}+3(-1)^{n}\left(\alpha^{n}-\beta^{n}\right)\right] \\
& =\frac{1}{\sqrt{5}}\left[\alpha^{3 n}-3 \alpha^{2 n} \beta^{n}+3 \alpha^{n} \beta^{2 n}-\beta^{3 n}+3(-1)^{n} \alpha^{n}-3(-1)^{n} \beta^{n}\right] \\
& =\frac{1}{\sqrt{5}}\left[\alpha^{3 n}-\beta^{3 n}-3\left(\alpha^{2} \beta\right)^{n}+3\left(\alpha \beta^{2}\right)^{n}+3(-1)^{n} \alpha^{n}-3(-1)^{n} \beta^{n}\right] \\
& =\frac{1}{\sqrt{5}}[\alpha^{3 n}-\beta^{3 n} \underbrace{-3(-\alpha)^{n}+3(-1)^{n} \alpha^{n}}_{\text {São cancelados para qualquer } n}+\underbrace{3(-\beta)^{n}-3(-1)^{n} \beta^{n}}_{\text {Também são cancelados }}] \\
& =\frac{1}{\sqrt{5}}\left[\alpha^{3 n}-\beta^{3 n}\right] \\
& =F_{3 n} .
\end{aligned}
$$

Demonstração de e):

Pelo item anterior, $F_{12 n}=5 F_{4 n}^{3}+3(-1)^{4 n} F_{4 n}=5 F_{4 n}^{3}+3 F_{4 n}$. Queremos mostrar que $3 F_{4 n} \mid F_{12 n}$.

Pelo Exemplo 2.9, $3 \mid F_{4 n}$. Assim $3 F_{4 n} \mid 5 F_{4 n}^{3}$. Dessa forma $3 F_{4 n} \mid 5 F_{4 n}^{3}+3 F_{4 n}$. Portanto $3 F_{4 n} \mid F_{12 n}$. 
Abaixo, apresentaremos um resultado sobre soma de quadrados de números de Fibonacci consecutivos.

Teorema 2.24. $F_{n-1}^{2}+F_{n}^{2}=F_{2 n-1}$ para todo $n$ natural.

Demonstração. Sabemos por (1.1) que $F_{n}=\left(\alpha^{n}-\beta^{n}\right) / \sqrt{5}$, onde $\alpha=(1+\sqrt{5}) / 2 \mathrm{e}$ $\beta=(1-\sqrt{5}) / 2$.

Observe que $\alpha+\beta=1, \alpha \beta=-1$ e $\alpha-\beta=\sqrt{5}$. Dessa forma:

$$
\begin{aligned}
F_{n-1}^{2}+F_{n}^{2} & =\frac{1}{5}\left(\alpha^{n}-\beta^{n}\right)^{2}+\frac{1}{5}\left(\alpha^{n-1}-\beta^{n-1}\right)^{2} \\
& =\frac{1}{5}\left[\alpha^{2 n}+\beta^{2 n}-2(\alpha \beta)^{n}+\alpha^{2 n-2}+\beta^{2 n-2}-2(\alpha \beta)^{n-1}\right] \\
& =\frac{1}{5}\left[\alpha^{2 n-1}\left(\alpha+\frac{1}{\alpha}\right)+\beta^{2 n-1}\left(\beta+\frac{1}{\beta}\right)\right] \\
& =\frac{1}{5}\left[\alpha^{2 n-1}(\alpha-\beta)+\beta^{2 n-1}(\beta-\alpha)\right] \\
& =\frac{1}{5}\left[\alpha^{2 n-1}(\sqrt{5})+\beta^{2 n-1}(-\sqrt{5})\right]=\frac{\sqrt{5}}{5}\left[\alpha^{2 n-1}-\beta^{2 n-1}\right] \\
& =\frac{1}{\sqrt{5}}\left(\alpha^{2 n-1}-\beta^{2 n-1}\right)=F_{2 n-1} .
\end{aligned}
$$

Portanto, $F_{n-1}^{2}+F_{n}^{2}=F_{2 n-1}, \forall n \in \mathbb{N}$.

Em 2010, Marques e Togbé demonstraram um teorema (ver [14]) envolvendo somas de $s$-ésimas potências de dois números de Fibonacci consecutivos. A motivação do estudo deles foi investigar se as somas de cubos, de quartas potências, de quintas potências, ..., de $s$-ésimas potências de dois números de Fibonacci consecutivos também eram números de Fibonacci. Eles mostraram que se $F_{n}^{s}+F_{n+1}^{s}$ é um número de Fibonacci para infinitos valores de $n$, então $s=1$ ou $s=2$.

Em 2011, Luca e Oyono resolveram completamente essa questão (ver [12]) mostrando que se $s>2$ e $n>2$ então $F_{n}^{s}+F_{n+1}^{s} \neq F_{m}$.

No Teorema 2.24, mostramos o caso em que $s=2$ usando a fórmula de Binet.

O teorema anterior afirma que a soma de dois quadrados de números de Fibonacci consecutivos é um número de Fibonacci. O leitor deve perceber que a Matemática envolvida na demonstração do teorema é completamente acessível ao Ensino Médio e, dessa forma, esse é mais um exemplo de questão que pode ser trabalhada em sala de aula para poder explorar, por exemplo, algumas propriedades algébricas. 


\section{CAPÍTULO 3}

\section{ORDEM DE APARIÇÃO NA SEQUENACIA DE FIBONACCI}

\subsection{Ordem de aparição na sequência de Fibonacci}

Nesse capítulo, definiremos a ordem de aparição de um número natural $n$ na sequência de Fibonacci, denotada por $z(n)$, e demonstraremos fórmulas fechadas para $z\left(F_{m} \pm 1\right)$.

Vamos mostrar que $z(n)$ está bem definida e, além disso, vamos caracterizar $z\left(L_{n}\right)$. Também demonstraremos que $z(p) \leq p+1$ para $p$ primo. Um resultado conhecido que não será abordado no trabalho, mas que tem a demonstração detalhada em [20], é que $z(n) \leq 2 n$ para qualquer $n$ natural. Essa cota superior para $z(n)$ é devida a Sallé ${ }^{1}$.

Outros assuntos também serão abordados, entretanto os citados acima são os mais relevantes.

Vimos até aqui várias propriedades das sequências de Fibonacci $\left(F_{n}\right)_{n \geq 0}$ e dos números de Lucas $\left(L_{n}\right)_{n \geq 0}$. Elas nos darão suporte para as demonstrações a seguir. Para prosseguir com o conteúdo, vamos introduzir o conceito principal deste capítulo.

Definição 3.1 (Ordem de aparição). Seja $n$ um número natural e $F_{m}$ o $m$-ésimo

\footnotetext{
${ }^{1}$ Em 1975, J. Sallé provou que $z(n) \leq 2 n$ para todo inteiro positivo $n$.
} 
número de Fibonacci. A ordem de aparição z(n) de um número natural $n$ na sequência de Fibonacci é o menor inteiro positivo $k$ tal que $n \mid F_{k}$.

Em outras palavras, se for conveniente para o entendimento, dado um número natural $n$ estamos interessados em descobrir o menor número de Fibonacci $F_{k}$ que é múltiplo de $n$ e após essa descoberta afirmar que o índice $k$ de tal número de Fibonacci é a ordem de aparição $z(n)$.

Para avançarmos no trabalho, detalhando a Definição 3.1, é essencial ter a sequência de Fibonacci em mente. Os 300 primeiros números de Fibonacci podem ser encontrados em [24].

O primeiro objetivo, em relação à ordem de aparição, é percorrer os 100 primeiros números naturais e obter as respectivas ordens de aparição na sequência de Fibonacci.

Lembrando quais são os 22 primeiros números de Fibonacci e com a definição de ordem de aparição, vamos listar alguns valores para exemplificar o que faremos.

\begin{tabular}{c|c|c|c|c|c|c|c|c|c|c|c}
\hline$n$ & 0 & 1 & 2 & 3 & 4 & 5 & 6 & 7 & 8 & 9 & 10 \\
\hline$F_{n}$ & 0 & 1 & 1 & 2 & 3 & 5 & 8 & 13 & 21 & 34 & 55 \\
\hline \hline$n$ & 11 & 12 & 13 & 14 & 15 & 16 & 17 & 18 & 19 & 20 & 21 \\
\hline$F_{n}$ & 89 & 144 & 233 & 377 & 610 & 987 & 1597 & 2584 & 4181 & 6765 & 10946 \\
\hline
\end{tabular}

Tabela 3.1: 22 primeiros números de Fibonacci

Temos, por exemplo, que $z(1)=1, z(2)=3, z(3)=4, z(4)=6, z(5)=5$, $z(6)=12, z(7)=8, z(8)=6, z(9)=12, z(10)=15, z(11)=10, z(12)=12$ e $z(13)=7$.

Os valores $z(n)$ listados acima foram todos calculados observando a Tabela 3.1 juntamente com os critérios de divisibilidade. Ao trabalhar em sala a sequência de Fibonacci, o professor pode definir ordem de aparição e com isso tratar de assuntos que não são do Ensino Médio, mas que precisam de conceitos básicos da Matemática para serem explorados.

Na Tabela 3.2 estão listadas a ordem de aparição na sequência de Fibonacci dos 100 primeiros números naturais. A partir dela, observaremos alguns padrões e faremos alguns questionamentos, que posteriormente serão explorados. A tabela seguinte pode ser encontrada em [6]. Lá também são encontradas outras conjecturas e demonstrações sobre o assunto. 


\begin{tabular}{|c|c||c|c||c|c||c|c||c|c|}
\hline$n$ & $z(n)$ & $n$ & $z(n)$ & $n$ & $z(n)$ & $n$ & $z(n)$ & $n$ & $z(n)$ \\
\hline \hline $\mathbf{1}$ & 1 & $\mathbf{2 1}$ & 8 & 41 & 20 & 61 & 15 & 81 & 108 \\
$\mathbf{2}$ & 3 & 22 & 30 & 42 & 24 & 62 & 30 & 82 & 60 \\
$\mathbf{3}$ & 4 & 23 & 24 & 43 & 44 & 63 & 24 & 83 & 84 \\
4 & 6 & 24 & 12 & 44 & 30 & 64 & 48 & 84 & 24 \\
$\mathbf{5}$ & 5 & 25 & 25 & 45 & 60 & 65 & 35 & 85 & 45 \\
6 & 12 & 26 & 21 & 46 & 24 & 66 & 60 & 86 & 132 \\
7 & 8 & 27 & 36 & 47 & 16 & 67 & 68 & 87 & 28 \\
$\mathbf{8}$ & 6 & 28 & 24 & 48 & 12 & 68 & 18 & 88 & 30 \\
9 & 12 & 29 & 14 & 49 & 56 & 69 & 24 & $\mathbf{8 9}$ & 11 \\
10 & 15 & 30 & 60 & 50 & 75 & 70 & 120 & 90 & 60 \\
11 & 10 & 31 & 30 & 51 & 36 & 71 & 70 & 91 & 56 \\
12 & 12 & 32 & 24 & 52 & 42 & 72 & 12 & 92 & 24 \\
$\mathbf{1 3}$ & 7 & 33 & 20 & 53 & 27 & 73 & 37 & 93 & 60 \\
14 & 24 & $\mathbf{3 4}$ & 9 & 54 & 36 & 74 & 57 & 94 & 48 \\
15 & 20 & 35 & 40 & $\mathbf{5 5}$ & 10 & 75 & 100 & 95 & 90 \\
$\mathbf{1 6}$ & 12 & 36 & 12 & 56 & 24 & 76 & 18 & 96 & 24 \\
$\mathbf{1 7}$ & 9 & 37 & 19 & 57 & 36 & 77 & 40 & 97 & 49 \\
$\mathbf{1 8}$ & 12 & 38 & 18 & 58 & 42 & 78 & 84 & 98 & 168 \\
$\mathbf{1 9}$ & 18 & 39 & 28 & 59 & 58 & 79 & 78 & 99 & 60 \\
20 & 30 & 40 & 30 & 60 & 60 & 80 & 60 & 100 & 150 \\
\hline
\end{tabular}

Tabela 3.2: Ordem de aparição dos 100 primeiros números naturais 
Observando a Tabela 3.2, alguns questionamentos podem ser feitos. Por exemplo:

- Quando $z(n)=n$ ?

- Para quais valores de $n, z(n)=2 n$ ?

- $z(n) \leq 2 n ?$

- Quando $F_{k}<n<F_{k+1}, z(n) \geq k+2$ ?

- Se $z(m)=z(n)=k$, então $z(\operatorname{mmc}(m, n))=k$ ?

- $z(p) \leq p+1$, para todo $p$ primo?

É importante notar que, pela Definição 3.1, se $n=F_{m}$, então $z(n)=z\left(F_{m}\right)=m$, $m \geq 3$. De fato, dado um número de Fibonacci $F_{m}, m \geq 3$, ele é o seu menor múltiplo natural.

Na Tabela 3.2, observamos que $z(1)=1, z(2)=3, z(3)=4, z(5)=5, z(8)=6$, $z(13)=7$ e assim sucessivamente. Desse modo $z\left(F_{m}\right)$ está bem definida.

Outra pergunta essencial é a seguinte: $z(n)$ está sempre definida? Ou seja, dado um número natural $n$ qualquer, sempre existirá um número de Fibonacci $F_{k}$ tal que $n \mid F_{k}$ ? Ou ainda, sendo $n$ natural existe um número de Fibonacci que é múltiplo de $n$ ? A seguir demonstraremos esse fato.

Teorema 3.2. Dado um número natural $n$ sempre existe um número de Fibonacci que é múltiplo de $n$ e, além disso, $z(n) \leq n^{2}+1, \forall n \geq 1$.

Demonstração. Seja $n \in \mathbb{N}$ e considere $S=\left\{\left(F_{k}, F_{k+1}\right)(\bmod n)\right\}_{k \in \mathbb{N}}$.

Como existem apenas $n$ resíduos módulo $n, S$ tem no máximo $n^{2}$ elementos distintos. Tomando $n^{2}+1$ elementos em $S$, teremos $\left(F_{m}, F_{m+1}\right) \equiv\left(F_{s}, F_{s+1}\right)(\bmod n)$ para alguns $m>s>0$. Em particular,

$$
F_{m+1} \equiv F_{s+1}(\bmod n) \text { e } F_{m} \equiv F_{s}(\bmod n) .
$$

Subtraindo essas congruências e usando a definição recursiva da sequência de Fibonacci, temos $F_{m-1} \equiv F_{s-1}(\bmod n)$. Repetindo esse processo $s$ vezes, obtemos

$$
F_{m-s} \equiv F_{s-s} \equiv F_{0} \equiv 0(\bmod n) \text {. }
$$


Observe que $m-s>0$ e, portanto, $n \mid F_{m-s}$. Utilizando a Proposição 2.6, temos que $n\left|F_{m-s}\right| F_{t \cdot(m-s)}$, para todo $t \in \mathbb{N}$, ou seja, $n$ divide infinitos números de Fibonacci. Portanto, pelo princípio da boa ordenação, existe o menor número de Fibonacci $F_{j}$ que é divisível por $n$. Note que $z(n) \leq n^{2}+1$.

Observe na Tabela 3.2 que alguns números naturais distintos têm a mesma ordem de aparição na sequência de Fibonacci. Por exemplo, $z(6)=z(9)=12=z(\operatorname{mmc}(6,9))=$ $z(18)=12$. Observe também que $z(45)=z(82)=z(99)=60$.

Será que podemos afirmar que $z(40590)=z(\operatorname{mmc}(45,82,99))=60$ ?

Com o teorema seguinte, vamos mostrar que quando duas ordens de aparição na sequência de Fibonacci, de dois números naturais distintos $m$ e $n$, são iguais a um número $k$, então a ordem de aparição na sequência de Fibonacci do menor múltiplo comum entre esses números $m$ e $n$ também é $k$.

Teorema 3.3. Se $z(m)=z(n)=k$, então $z(\operatorname{mmc}(m, n))=k$.

Demonstração. Suponha que $z(m)=z(n)=k$, então, por definição, $m \mid F_{k}$ e $n \mid F_{k}$. $\operatorname{Logo} \operatorname{mmc}(m, n) \mid F_{k}$. Suponha também que $\operatorname{mmc}(m, n) \mid F_{j} \operatorname{com} j<k$, então $m \mid F_{j}$ para $j<k$. Contudo, uma vez que $z(m)=k$, por hipótese, a relação seguinte $\operatorname{mmc}(m, n) \mid F_{j} \operatorname{com} j<k$ é impossível. Portanto, $k$ é o menor inteiro tal que $\operatorname{mmc}(m, n) \mid F_{k}$, ou seja, $z(\operatorname{mmc}(m, n))=k$.

O resultado anterior pode ser estendido para mais de dois fatores. Com isso concluímos que $z(40590)=60$, ou seja, $F_{60}$ é múltiplo de 40.590 e, além disso, $F_{60}=1.548 .008 .755 .920$ é o menor número de Fibonacci que é múltiplo de 40.590.

Conhecendo o Teorema 3.3 é possível calcular, com base na Tabela 3.2, outras ordens de aparição na sequência de Fibonacci. Por exemplo, $z(96)=z(92)=24$, portanto $z(\operatorname{mmc}(92,96))=z(2208)=24$.

Dessa forma, uma atividade que pode ser sugerida em projetos extra classe é justamente explorar outros resultados sobre a ordem de aparição na sequência de Fibonacci. Esse exercício trabalhará, entre outros fatos, os conceitos básicos de divisibilidade e menor múltiplo comum.

O corolário a seguir leva em consideração o fato de dois números consecutivos serem primos entre si. 
Corolário 3.4. Se $z(n)=z(n+1)=k$, então $z(n(n+1))=k$.

Demonstração. Segue diretamente do Teorema 3.3 e do seguinte fato:

$$
\operatorname{mmc}(n, n+1)=n(n+1) .
$$

Observe que o corolário anterior tem como hipótese um fato nada trivial. Ele considera que existe solução para a equação Diofantina $z(n)=z(n+1)$. Porém, quais as condições para que $z(n)=z(n+1)$ ? Alguns matemáticos conjecturaram $z(n) \neq z(n+1)$ para qualquer número natural $n$ (ver [6]), porém isso é falso. A demonstração de infinitas soluções para $z(n)=z(n+1)$ já foi realizada por Luca e Pomerance (ver [13]).

O objetivo principal desse capítulo é mostrar que existem infinitas soluções para $z(n)=z(n+2)$, mas, antes disso, precisamos de outros resultados auxiliares.

A seguir vamos tratar de quatro proposições envolvendo números de Fibonacci, números de Lucas e ordem de aparição na sequência de Fibonacci. Esses resultados darão suporte à demonstração do teorema que define fórmulas fechadas para $z\left(F_{m} \pm 1\right)$.

Proposição 3.5. Se $F_{n} \mid m$, então $n \mid z(m)$.

Demonstração. Por hipótese, $F_{n} \mid m$ e, por definição, $m \mid F_{z(m)}$. Logo, $F_{n} \mid F_{z(m)}$. Assim, pelo Corolário 2.8, $n \mid z(m)$.

Proposição 3.6. Se $L_{n} \mid m$, então $2 n \mid z(m)$.

Demonstração. Observe que $L_{n} \mid m$ e $m \mid F_{z(m)}$, portanto $L_{n} \mid F_{z(m)}$. Pelo Teorema $2.23 a), L_{n}\left|F_{z(m)} \Leftrightarrow n\right| z(m)$ e $z(m) / n$ é par. Portanto, $2 n \mid z(m)$.

Proposição 3.7. Se $n \mid F_{m}$, então $z(n) \mid m$.

Demonstração. No intuito de provar a afirmação, seja $m=z(n) \cdot q+r$, onde $q$ e $r$ são inteiros, com $0 \leq r<z(n)$. Portanto, pelo Corolário 2.5, obtemos:

$$
(-1)^{z(n) \cdot q} F_{r}=F_{m} F_{z(n)+1}-F_{z(n)} F_{m+1} .
$$

Observe que, por hipótese, $n$ divide $F_{m}$ e, por definição, $n \mid F_{z(n)}$. Assim, $n$ divide qualquer combinação linear inteira entre $F_{m}$ e $F_{z(n)}$, em especial, 


$$
n \mid F_{m} F_{z(n)+1}-F_{z(n)} F_{m+1},
$$

ou seja, $n \mid F_{r}$. Implicando $r=0$, pois $r<z(n)$. Logo, $m=z(n) \cdot q$, ou seja, $z(n) \mid m$.

Proposição 3.8. Se $a z(m)=b z(n)$, então $\max \{z(m), z(n)\}|z(\operatorname{mmc}(m, n))| a z(m)$, para $a, b \in \mathbb{N}$.

Demonstração. Sabemos que $n \mid F_{z(n)}$. Pela Proposição 2.6, $F_{z(n)} \mid F_{b z(n)}$. Logo, $n \mid F_{a z(m)}=F_{b z(n)}$.

Por outro lado, $m\left|F_{z(m)}\right| F_{a z(m)}$. Uma vez que $n \mid F_{a z(m)}$ e $m \mid F_{a z(m)}$ temos que $\operatorname{mmc}(m, n) \mid F_{a z(m)}$. Dessa forma, pela proposição anterior, $z(\operatorname{mmc}(m, n))$ divide $a z(m)$. Além disso, observe que $\max \{m, n\}|\operatorname{mmc}(m, n)| F_{z(\operatorname{mmc}(\mathrm{m}, \mathrm{n}))}$. Portanto, pela Proposição 3.7, $\max \{z(m), z(n)\}|z(\operatorname{mmc}(m, n))| a z(m)$.

Ainda com base na Tabela 3.2, observamos que entre os 25 números primos $p$ listados a ordem de aparição $z(p) \leq p+1$. Por exemplo, $z(5)=5, z(7)=8, z(11)=$ $10, z(13)=7, z(23)=24, z(31)=30, z(43)=44, z(83)=84$ e $z(97)=49$. Esse fato é estendido para todos os números primos facilmente. Isso é o que mostraremos na proposição seguinte.

Proposição 3.9. Para todo primo $p$, vale que $z(p) \leq p+1$.

Demonstração. Sabemos pelo Corolário 2.20 que $p \mid F_{p-\left(\frac{p}{5}\right)}$. Logo pela Proposição 3.7, $z(p) \mid p-\left(\frac{p}{5}\right) . \operatorname{Assim} z(p) \leq p-\left(\frac{p}{5}\right) \leq p+1$.

Ao analisar a Tabela 3.2 é possível perceber que $z\left(F_{m}-1\right)>z\left(F_{m}\right)<z\left(F_{m}+1\right)$ para os valores de $F_{m}(m \geq 5)$ listados. Logo abaixo vamos mostrar que esse é um resultado geral. Para isso o leitor deve lembrar que, pela Definição 3.1, se $n=F_{m}$ é um número de Fibonacci, então $z(n)=z\left(F_{m}\right)=m, m \geq 3$.

Proposição 3.10. $z\left(F_{m}-1\right)>z\left(F_{m}\right)<z\left(F_{m}+1\right)$ para $m \geq 5$.

Demonstração. Seja $z\left(F_{m}-1\right)=k$. Então, pela Definição $3.1, F_{m}-1$ divide $F_{k}$, ou seja, existe um inteiro $a \geq 2$ (pois não existem números consecutivos de Fibonacci maiores que 3$)$ tal que $F_{k}=a\left(F_{m}-1\right)$. Assim, $F_{k}>F_{m}$, pois $a \geq 2$, o que implica $k>m=z\left(F_{m}\right)$. Portanto, $z\left(F_{m}-1\right)>z\left(F_{m}\right)$. 
Analogamente, suponha que $z\left(F_{m}+1\right)=l$, então $F_{m}+1$ divide $F_{l}$ e, portanto, $F_{m}+1 \leq F_{l}$. Isso implica $F_{m}<F_{l}$, ou seja, $m=z\left(F_{m}\right)<l$. Assim, $z\left(F_{m}\right)<z\left(F_{m}+1\right)$. Dessa maneira, $z\left(F_{m}-1\right)>z\left(F_{m}\right)<z\left(F_{m}+1\right)$.

Em um artigo recente (ver [15]), Marques provou que existem infinitos números naturais $n$ que não são números de Fibonacci, porém $z(n \pm 1)>z(n)$, ou seja, esses números naturais se comportam como números de Fibonacci.

A seguir vamos mostrar que dado um número natural $n$ entre dois números de Fibonacci $F_{k}$ e $F_{k+1}$, então $z(n) \geq k+2$.

Proposição 3.11. $z(n) \geq k+2$ para todo $n$ pertencente ao intervalo $\left(F_{k}, F_{k+1}\right)$.

Demonstração. Por hipótese, $n>3$ é um número natural tal que $F_{k}<n<F_{k+1}$. Suponha que $z(n)=j$. Pela Definição $3.1, n \mid F_{j}$, ou seja, $F_{j}=a \cdot n$ para algum inteiro a. Por outro lado $F_{k+1}=F_{k}+F_{k-1} \leq 2 F_{k}$. Uma vez que $a \neq 1$, pois $n$ não é um número de Fibonacci, segue que $a \geq 2$. Portanto $F_{j}>n>F_{k}$. Assim $F_{j}=a \cdot n>2 F_{k} \geq F_{k+1}$. Logo $F_{j}>F_{k+1}$, ou seja, $j=z(n)>k+1$. Portanto, $z(n) \geq k+2$.

\subsection{Infinitas soluções para $z(n)=z(n+2)$ e fórmulas}

\section{fechadas para $z\left(F_{m} \pm 1\right)$}

Sabendo que a ordem de aparição na sequência de Fibonacci está bem definida, podemos pensar em encontrar fórmulas fechadas para $z(n)$.

O objetivo desta seção é demonstrar que existem infinitas soluções para $z(n)=$ $z(n+2)$ e caracterizar $z\left(F_{m} \pm 1\right)$. Para isso precisaremos olhar para $m$ módulo 4 . Desse modo, detalharemos cada um dos casos: $z\left(F_{4 m} \pm 1\right), z\left(F_{4 m+1} \pm 1\right), z\left(F_{4 m+2} \pm 1\right)$ e $z\left(F_{4 m+3} \pm 1\right)$.

O caso $z\left(F_{4 m+2} \pm 1\right)$ será subdividido em outros dois, a saber: $z\left(F_{8 m+2} \pm 1\right)$ e $z\left(F_{8 m+6} \pm 1\right)$, devido à equivalência das classes de restos na divisão de $m$ por 4 e por 8 , respectivamente.

Em uma parte da demonstração do Teorema 3.14 precisaremos da definição da ordem $p$-ádica de um número natural, assim como precisaremos de um resultado que descreve a ordem $p$-ádica de números de Fibonacci. Vamos nos limitar a citar o teorema 
sem realizar a demonstração. A prova completa do Teorema 3.13, sobre a ordem $p$ ádica, pode ser encontrada em [9].

Definição 3.12. A ordem p-ádica de um número natural $r$, denotada por $\nu_{p}(r)$, é o expoente da maior potência de um primo p que divide $r$.

A ordem $p$-ádica de um número de Fibonacci foi completamente caracterizada, mas para os nossos objetivos é suficiente o teorema seguinte.

Teorema 3.13. Para $n \geq 1$, temos:

$$
\begin{aligned}
& \nu_{2}\left(F_{n}\right)=\left\{\begin{array}{cl}
0, & \text { se } n \equiv 1,2(\bmod 3) ; \\
1, & \text { se } n \equiv 3(\bmod 6) ; \\
3, & \text { se } n \equiv 6(\bmod 12) ; \\
\nu_{2}(n)+2, & \text { se } n \equiv 0(\bmod 12) .
\end{array}\right. \\
& \nu_{3}\left(F_{n}\right)=\left\{\begin{array}{cc}
\nu_{3}(n)+1, & \text { se } n \equiv 0(\bmod 4) ; \\
0, & \text { caso contrário. }
\end{array}\right.
\end{aligned}
$$

Com as breves considerações iniciais acima, e tendo como base alguns resultados abordados nesse texto, estamos prontos para demonstrar o principal teorema desse trabalho. Observe que além de caracterizar $z\left(F_{m} \pm 1\right)$ o teorema seguinte demonstra que existem infinitas soluções para $z(n)=z(n+2)$.

Teorema 3.14. Temos:
a) $z\left(F_{4 m} \pm 1\right)=2\left(4 m^{2}-1\right)$, se $m>1$;
b) $2 \cdot z\left(F_{4 m+1}-1\right)=z\left(F_{4 m+1}+1\right)=4 m(2 m+1)$, se $m \geq 1$;
c.1) $z\left(F_{8 m+2}+1\right)=8 m(2 m+1)$, se $m \geq 1$;
c.2) $z\left(F_{8 m+2}-1\right)=12 m(2 m+1)$, se $m \geq 1$;
c.3) $z\left(F_{8 m+6}+1\right)=12(m+1)(2 m+1)$, se $m \geq 0$;
c.4) $z\left(F_{8 m+6}-1\right)=8(m+1)(2 m+1)$, se $m \geq 0$;
d) $2 \cdot z\left(F_{4 m+3}-1\right)=z\left(F_{4 m+3}+1\right)=4(m+1)(2 m+1)$, se $m \geq 1$. 
Demonstração. Em primeiro lugar, observe que a Proposição 2.12 fornece imediatamente fatorações para $F_{m} \pm 1$, dependendo da classe de $m$ módulo $4: F_{m} \pm 1=F_{a} \cdot L_{b}$, onde $2 a, 2 b \in\{m \pm 1, m \pm 2\}$.

Para demonstrar $a$ ), considere, na Proposição 2.12, $a=2 m+\varepsilon_{1}$ e $b=2 m+\varepsilon_{2}$, onde $\varepsilon_{1}, \varepsilon_{2} \in\{ \pm 1\}$ são distintos. Assim, obtemos:

$$
F_{2 m+\varepsilon_{1}} \cdot L_{2 m+\varepsilon_{2}}=F_{4 m} \pm 1 \text {. }
$$

Note, pelo Exemplo 2.1, que

$$
F_{2\left(2 m+\varepsilon_{1}\right)\left(2 m+\varepsilon_{2}\right)}=F_{\left(2 m+\varepsilon_{1}\right)\left(2 m+\varepsilon_{2}\right)} \cdot L_{\left(2 m+\varepsilon_{1}\right)\left(2 m+\varepsilon_{2}\right)}
$$

e note também, pela Proposição 2.6 e pelo Teorema 2.23 b), respectivamente, que

$$
F_{2 m+\varepsilon_{1}} \mid F_{\left(2 m+\varepsilon_{1}\right)\left(2 m+\varepsilon_{2}\right)} \text { e } L_{2 m+\varepsilon_{2}} \mid L_{\left(2 m+\varepsilon_{1}\right)\left(2 m+\varepsilon_{2}\right)} .
$$

Assim, $F_{4 m} \pm 1=F_{2 m+\varepsilon_{1}} \cdot L_{2 m+\varepsilon_{2}} \mid F_{\left(2 m+\varepsilon_{1}\right)\left(2 m+\varepsilon_{2}\right)} \cdot L_{\left(2 m+\varepsilon_{1}\right)\left(2 m+\varepsilon_{2}\right)}$. Sabemos que

$$
F_{\left(2 m+\varepsilon_{1}\right)\left(2 m+\varepsilon_{2}\right)} \cdot L_{\left(2 m+\varepsilon_{1}\right)\left(2 m+\varepsilon_{2}\right)}=F_{2\left(2 m+\varepsilon_{1}\right)\left(2 m+\varepsilon_{2}\right)}=F_{2\left(4 m^{2}-1\right)},
$$

$\operatorname{logo} F_{4 m} \pm 1 \mid F_{2\left(4 m^{2}-1\right)}$. Portanto, pela Proposição 3.7, $z\left(F_{4 m} \pm 1\right) \mid 2\left(4 m^{2}-1\right)$.

Dessa maneira,

$$
z\left(F_{4 m} \pm 1\right) \leq 2\left(4 m^{2}-1\right)
$$

Por outro lado, ambos $F_{2 m+\varepsilon_{1}}$ e $L_{2 m+\varepsilon_{2}}$ dividem $F_{4 m} \pm 1$. Logo, pela Proposição 3.5 , obtemos que $2 m+\varepsilon_{1} \mid z\left(F_{4 m} \pm 1\right)$ e, pela Proposição 3.6 , temos que $2\left(2 m+\varepsilon_{2}\right)$ divide $z\left(F_{4 m} \pm 1\right)$.

Uma vez que $\operatorname{mdc}\left(2 m+\varepsilon_{1}, 2\left(2 m+\varepsilon_{2}\right)\right)=1$, então $2\left(4 m^{2}-1\right) \mid z\left(F_{4 m} \pm 1\right)$. Dessa forma, $z\left(F_{4 m} \pm 1\right) \geq 2\left(4 m^{2}-1\right)$.

Observando que $2\left(4 m^{2}-1\right) \leq z\left(F_{4 m} \pm 1\right) \leq 2\left(4 m^{2}-1\right)$ temos a igualdade desejada, isto é, $z\left(F_{4 m} \pm 1\right)=2\left(4 m^{2}-1\right)$, se $m>1$.

Na demonstração de $b$ ) e $d$ ) façamos, na Proposição 2.12, $a=2 m+\varepsilon_{1}$ e $b=2 m+\varepsilon_{2}$, onde $\varepsilon_{1}, \varepsilon_{2} \in\{ \pm 1\}$ são distintos, e $\delta \in\{0,2\}$. Dessa forma,

$$
F_{2 m+1} \cdot L_{2 m+\delta}=F_{4 m+1+\delta}+1 \text { e } F_{2 m+\delta} \cdot L_{2 m+1}=F_{4 m+1+\delta}-1 .
$$

Vamos considerar separadamente os casos $F_{4 m+1+\delta}+1$ e $F_{4 m+1+\delta}-1$. 


\section{O caso: $F_{4 m+1+\delta}+1$.}

Observe, pelo Exemplo 2.1, que

$$
F_{2(2 m+1)(2 m+\delta)}=F_{(2 m+1)(2 m+\delta)} \cdot L_{(2 m+1)(2 m+\delta)} .
$$

Além disso, note, pela Proposição 2.6, que $F_{2 m+1} \mid F_{(2 m+1)(2 m+\delta)}$ e note também que, pelo Teorema $2.23 b), L_{2 m+\delta} \mid L_{(2 m+1)(2 m+\delta)}$. Assim,

$$
F_{2 m+1} \cdot L_{2 m+\delta}=F_{4 m+1+\delta}+1 \mid F_{(2 m+1)(2 m+\delta)} \cdot L_{(2 m+1)(2 m+\delta)}=F_{2(2 m+1)(2 m+\delta)},
$$

ou seja, $F_{4 m+1+\delta}+1 \mid F_{2(2 m+1)(2 m+\delta)}$.

Portanto, pela Proposição 3.7,

$$
z\left(F_{4 m+1+\delta}+1\right) \mid 2(2 m+1)(2 m+\delta)
$$

e, dessa forma,

$$
z\left(F_{4 m+1+\delta}+1\right) \leq 2(2 m+1)(2 m+\delta) .
$$

Por outro lado, ambos $F_{2 m+1}$ e $L_{2 m+\delta}$ dividem $F_{4 m+1+\delta}+1$. Logo, pela Proposição 3.5 , temos que $2 m+1 \mid z\left(F_{4 m+1+\delta}+1\right)$ e, pela Proposição 3.6, obtemos que $2(2 m+\delta)$ divide $z\left(F_{4 m+1+\delta}+1\right)$.

Uma vez que o $\operatorname{mdc}(2 m+1,2(2 m+\delta))=1$, temos que

$$
2(2 m+1)(2 m+\delta) \mid z\left(F_{4 m+1+\delta}+1\right),
$$

de onde segue que

$$
2(2 m+1)(2 m+\delta) \leq z\left(F_{4 m+1+\delta}+1\right) .
$$

Levando em conta a desigualdade

$$
2(2 m+1)(2 m+\delta) \leq z\left(F_{4 m+1+\delta}+1\right) \leq 2(2 m+1)(2 m+\delta),
$$

temos que $z\left(F_{4 m+1+\delta}+1\right)=2(2 m+1)(2 m+\delta)$.

Agora basta considerar os valores de $\delta$.

- Se $\delta=0$, então

$$
z\left(F_{4 m+1}+1\right)=2(2 m+1)(2 m)=4 m(2 m+1),
$$

finalizando o caso positivo de $b$ ).

- Se $\delta=2$, então

$$
z\left(F_{4 m+3}+1\right)=2(2 m+1)(2 m+2)=4(m+1)(2 m+1),
$$

o que demonstra a parte positiva de $d$ ). 


\section{O caso: $F_{4 m+1+\delta}-1$.}

O caso $F_{4 m+1+\delta}-1$ tem uma análise diferente, porque $L_{2 m+1} \nmid L_{(2 m+1)(2 m+\delta)}$ uma vez que $(2 m+1)(2 m+\delta) /(2 m+1)$ é par, conforme o Teorema $2.23 b)$.

Note, pelo Teorema $2.23 a$ ), que

$$
L_{2 m+1} \mid F_{(2 m+1)(2 m+\delta)} .
$$

Além disso, note, pela Proposição 2.6 , que

$$
F_{2 m+\delta} \mid F_{(2 m+1)(2 m+\delta)}
$$

e, observe também, pelo Teorema 2.23 c), que $\operatorname{mdc}\left(F_{2 m+\delta}, L_{2 m+1}\right)=1$. Assim,

$$
L_{2 m+1} \cdot F_{2 m+\delta} \mid F_{(2 m+1)(2 m+\delta)} .
$$

Mas $F_{2 m+\delta} \cdot L_{2 m+1}=F_{4 m+1+\delta}-1$, de modo que

$$
\left(F_{4 m+1+\delta}-1\right) \mid F_{(2 m+1)(2 m+\delta)},
$$

de onde segue, pela Proposição 3.7, que $z\left(F_{4 m+1+\delta}-1\right) \mid(2 m+1)(2 m+\delta)$, fornecendo $z\left(F_{4 m+1+\delta}-1\right) \leq(2 m+1)(2 m+\delta)$.

Por outro lado, vimos que $F_{2 m+\delta} \cdot L_{2 m+1}=F_{4 m+1+\delta}-1$, ou seja, ambos $F_{2 m+\delta}$ e $L_{2 m+1}$ dividem $F_{4 m+1+\delta}-1$. Observe, pela Proposição 3.5, que

$$
2 m+\delta \mid z\left(F_{4 m+1+\delta}-1\right) \text {. }
$$

Observe também, pela Proposição 3.6, que $2(2 m+1) \mid z\left(F_{4 m+1+\delta}-1\right)$.

Visto que o $\operatorname{mdc}(2 m+1,2 m+\delta)=1$, temos que $(2 m+1)(2 m+\delta) \mid z\left(F_{4 m+1+\delta}-1\right)$, ou seja $(2 m+1)(2 m+\delta) \leq z\left(F_{4 m+1+\delta}-1\right)$. Das duas desigualdades encontradas segue o resultado $z\left(F_{4 m+1+\delta}-1\right)=(2 m+1)(2 m+\delta)$.

- Se $\delta=0$, então $z\left(F_{4 m+1}-1\right)=(2 m)(2 m+1)$, o que equivale a

$$
2 \cdot z\left(F_{4 m+1}-1\right)=4 m(2 m+1),
$$

mostrando o caso negativo de $b$ ).

- Se $\delta=2$, então

$$
z\left(F_{4 m+3}-1\right)=(2 m+1)(2 m+2)=2(m+1)(2 m+1),
$$

o que é equivalente a parte negativa de $d$ ).

Agora vamos mostrar a letra $c$ ). Vamos dividir a demonstração em duas partes. Primeiramente vamos mostrar os itens c.1) e c.4) e, em seguida, vamos demonstrar os casos $c .2$ ) e $c .3)$. 


\section{Os casos: $F_{8 m+2}+1$ e $F_{8 m+6}-1$.}

Seja $\delta \in\{0,4\}$. Pela Proposição 2.12, com $a=4 m+2$ e $b=4 m+\delta$, temos: $F_{4 m+2} \cdot L_{4 m+\delta}=F_{8 m+2+\delta}+(-1)^{4 m+\delta} \cdot F_{2-\delta}$, o que pode ser reescrito como

$$
F_{4 m+2} \cdot L_{4 m+\delta}=F_{8 m+2+\delta}+(-1)^{\delta / 4}
$$

uma vez que $F_{-2}=-1$ e $(-1)^{4 m+\delta}$ é positivo.

Note, pela Proposição 2.6, que $F_{4 m+2}$ divide $F_{(4 m+2)(4 m+\delta)}$ e que $L_{4 m+\delta}$ também divide $F_{(4 m+2)(4 m+\delta)}=F_{2(2 m+1)(4 m+\delta)}$, pelo Teorema $\left.2.23 a\right)$.

Por outro lado, observe que $d=\operatorname{mdc}(4 m+2,4 m+\delta)=2,(4 m+2) / d=2 m+1$ (ímpar) e $(4 m+\delta) / d=(4 m+\delta) / 2=2(m+\delta / 4)$ (par). Portanto, o Teorema $2.23 \mathrm{c})$ implica $\operatorname{mdc}\left(F_{4 m+2}, L_{4 m+\delta}\right)=1$ ou 2 . Vamos mostrar que $\operatorname{mdc}\left(F_{4 m+2}, L_{4 m+\delta}\right)=1$.

Suponha que ambos $F_{4 m+2}$ e $L_{4 m+\delta}$ sejam pares. Assim, os Exemplos 2.9 e 2.10 garantem respectivamente que 3 divide $4 m+2$ e 3 divide $4 m+\delta$. Desse modo, 3 divide qualquer combinação linear entre $4 m+2$ e $4 m+\delta$, como por exemplo, $3 \mid(\delta-2) \in\{ \pm 2\}$. Mas isto é um absurdo! Logo, $\operatorname{mdc}\left(F_{4 m+2}, L_{4 m+\delta}\right) \neq 2$, ou seja, $\operatorname{mdc}\left(F_{4 m+2}, L_{4 m+\delta}\right)=1$.

Sabendo que $F_{4 m+2}$ e $L_{4 m+\delta}$ são primos entre si, podemos afirmar que

$$
F_{8 m+2+\delta}+(-1)^{\delta / 4}=F_{4 m+2} \cdot L_{4 m+\delta} \mid F_{2(2 m+1)(4 m+\delta)} .
$$

Portanto, pelo Teorema 3.7, $z\left(F_{8 m+2+\delta}+(-1)^{\delta / 4}\right) \mid 2(2 m+1)(4 m+\delta)$, o que implica $z\left(F_{8 m+2+\delta}+(-1)^{\delta / 4}\right) \leq 2(2 m+1)(4 m+\delta)$.

Para a desigualdade oposta, observe que

$$
F_{2 m+1}\left|F_{2(2 m+1)}=F_{4 m+2}\right| F_{8 m+2+\delta}+(-1)^{\delta / 4} .
$$

Assim, pelo Teorema 3.5, $2 m+1 \mid z\left(F_{8 m+2+\delta}+(-1)^{\delta / 4}\right)$. Observe também que $L_{4 m+\delta}$ divide $F_{8 m+2+\delta}+(-1)^{\delta / 4}$ e assim, pelo Teorema 3.6 , obtemos que $2(4 m+\delta)$ divide $z\left(F_{8 m+2+\delta}+(-1)^{\delta / 4}\right)$.

Novamente usamos o fato que $\operatorname{mdc}(2 m+1,2(4 m+\delta))=1$ fornecendo que $2(2 m+1)(4 m+\delta) \mid z\left(F_{8 m+2+\delta}+(-1)^{\delta / 4}\right)$. Logo,

$$
2(2 m+1)(4 m+\delta) \leq z\left(F_{8 m+2+\delta}+(-1)^{\delta / 4}\right) .
$$

Assim, obtemos a igualdade $z\left(F_{8 m+2+\delta}+(-1)^{\delta / 4}\right)=2(2 m+1)(4 m+\delta)$.

- Se $\delta=0$, temos $z\left(F_{8 m+2}+1\right)=2(2 m+1)(4 m)=8 m(2 m+1), m \geq 1$, como queríamos.

- Se $\delta=4$, temos $z\left(F_{8 m+6}-1\right)=2(2 m+1)(4 m+4)=8(m+1)(2 m+1), m \geq 0$, e a relação está provada. 


\section{$\underline{\text { Os casos } F_{8 m+2}-1 \text { e } F_{8 m+6}+1}$.}

Seja $\delta \in\{0,4\}$. Pela Proposição 2.12, com $a=4 m+\delta$ e $b=4 m+2$, temos: $F_{4 m+\delta} \cdot L_{4 m+2}=F_{8 m+2+\delta}+(-1)^{4 m+2} \cdot F_{\delta-2}=F_{8 m+2+\delta}+(-1)^{(\delta-4) / 4}$. Note que ambos $F_{4 m+\delta}$ e $L_{4 m+2}$ dividem $F_{(4 m+\delta)(2 m+1)}$, pela Proposição 2.6 e pelo Teorema $2.23 \mathrm{a}$ ), respectivamente.

Observe que $\operatorname{mdc}(4 m+\delta, 4 m+2)=2,(4 m+\delta) / 2=2(m+\delta / 4)$ é par e $(4 m+2) / 2=2 m+1$ é ímpar. Então, pelo Teorema $2.23 \mathrm{c})$,

$$
\operatorname{mdc}\left(F_{4 m+\delta}, L_{4 m+2}\right)=L_{\mathrm{mdc}(4 m+\delta, 4 m+2)}=L_{2}=3 .
$$

Dessa forma, $F_{4 m+\delta} \cdot L_{4 m+2} \mid 3 F_{(4 m+\delta)(2 m+1)}$. Observe que $(4 m+\delta)(2 m+1)$ é múltiplo de 4, assim, pelo Teorema $2.23 \mathrm{e}), 3 F_{(4 m+\delta)(2 m+1)} \mid F_{3(4 m+\delta)(2 m+1)}$.

Isso implica $\left(F_{8 m+2+\delta}+(-1)^{(\delta-4) / 4}\right) \mid F_{3(4 m+\delta)(2 m+1)}$. Portanto, pelo Teorema 3.7,

$$
z\left(F_{8 m+2+\delta}+(-1)^{(\delta-4) / 4}\right) \mid 3(4 m+\delta)(2 m+1) .
$$

Por outro lado, a fatoração de $F_{8 m+2+\delta}+(-1)^{(\delta-4) / 4}$ fornece que $(4 m+\delta)(2 m+1)$ divide $z\left(F_{8 m+2+\delta}+(-1)^{(\delta-4) / 4}\right)$. Assim, podemos concluir que:

$z\left(F_{8 m+2+\delta}+(-1)^{(\delta-4) / 4}\right) \in\{(4 m+\delta)(2 m+1), 3(4 m+\delta)(2 m+1)\}$ com $m \geq 1$.

Agora é suficiente provar que $z\left(F_{8 m+2+\delta}+(-1)^{(\delta-4) / 4}\right) \nmid F_{(4 m+\delta)(2 m+1)}$. Provaremos utilizando redução ao absurdo.

Suponha que exista um inteiro $t$ tal que $F_{(4 m+\delta)(2 m+1)}=t\left(F_{8 m+2+\delta}+(-1)^{(\delta-4) / 4}\right)$.

Fatorando o lado direito da igualdade anterior obtemos:

$$
F_{(4 m+\delta)(2 m+1)}=t \cdot F_{4 m+\delta} \cdot L_{4 m+2} .
$$

Tendo em vista o Exemplo 2.1 e multiplicando a última igualdade por $F_{4 m+2}$, temos:

$$
F_{(4 m+\delta)(2 m+1)} \cdot F_{4 m+2}=t \cdot F_{4 m+\delta} \cdot L_{4 m+2} \cdot F_{4 m+2}=t \cdot F_{4 m+\delta} \cdot F_{8 m+4},
$$

o que fornece $\nu_{3}\left(F_{(4 m+\delta)(2 m+1)}\right) \geq \nu_{3}\left(F_{4 m+\delta} \cdot F_{8 m+4}\right)$. Entretanto, o Teorema 3.13 afirma que $\nu_{3}\left(F_{(4 m+\delta)(2 m+1)}\right)=\nu_{3}((4 m+\delta)(2 m+1))+1$, enquanto $\nu_{3}\left(F_{4 m+\delta} \cdot F_{8 m+4}\right)=$ $\nu_{3}\left(F_{4 m+\delta}\right)+\nu_{3}\left(F_{8 m+4}\right)=\nu_{3}(4 m+\delta)+1+\nu_{3}(8 m+4)+1=\nu_{3}((4 m+\delta)(2 m+1))+2$, ou seja, $\nu_{3}\left(F_{(4 m+\delta)(2 m+1)}\right)<\nu_{3}\left(F_{4 m+\delta} \cdot F_{8 m+4}\right)$. O que é um absurdo!

Assim, $\left(F_{8 m+2+\delta}+(-1)^{(\delta-4) / 4}\right) \nmid F_{(4 m+\delta)(2 m+1)}$.

Portanto, obtemos que

$$
z\left(F_{8 m+2+\delta}+(-1)^{(\delta-4) / 4}\right)=3(4 m+\delta)(2 m+1) .
$$


Para terminar a demonstração, observe que

- Se $\delta=0$ então $z\left(F_{8 m+2}-1\right)=3(4 m)(2 m+1)=12 m(2 m+1), m \geq 1$.

- Se $\delta=4$, temos $z\left(F_{8 m+6}+1\right)=3(4 m+4)(2 m+1)=12(m+1)(2 m+1), m \geq 0$.

De posse do resultado anterior, podemos verificar para alguns valores de $m$, diretamente pelas fórmulas deduzidas, a ordem de aparição na sequência de Fibonacci de alguns números naturais encontrados na Tabela 3.2.

Por exemplo, para $m=0,1$ ou 2, encontramos os seguintes valores: $z(4)=6$, $z(6)=12, z(7)=8, z(9)=12, z(12)=12, z(14)=24, z(20)=z(22)=30$, $z(33)=20, z(35)=40, z(54)=36, z(56)=24, z(88)=30$ e $z(90)=60$.

Se observarmos atentamente a afirmação em $a$ ), do Teorema 3.14, perceberemos que existem infinitos valores de $n$ que são soluções da equação $z(n)=z(n+2)$, a saber $n=F_{4 m}-1, \forall m>1$. Vimos, por exemplo, que $z(20)=z(22)=30$.

Luca e Pomerance mostraram recentemente (ver[13]) que existem infinitas soluções para a equação Diofantina $z(n)=z(n+1)$. Nesse sentido, uma pergunta em aberto, até a publicação desse trabalho, é se existe solução para $z(n)=z(n+1)=z(n+2)$.

Corolário 3.15. Para $m \geq 1$, temos que $z\left(F_{12 m}^{2}-1\right)=2\left(36 m^{2}-1\right)$.

Demonstração. Pelo Teorema 3.14, $z(n)=z(n+2)=2\left(36 m^{2}-1\right)$, onde $n=F_{12 m}-1$. Observe que $\operatorname{mmc}(n, n+2)=n(n+2)$, uma vez que $n$ é ímpar.

Pelo Teorema 3.3, temos que $z(n)=z(n+2)=z(\operatorname{mmc}(n, n+2))=z(n(n+2))=$ $z\left(\left(F_{12 m}-1\right)\left(F_{12 m}+1\right)\right)=z\left(F_{12 m}^{2}-1\right)=2\left(36 m^{2}-1\right)$.

Para encerrar esse capítulo, temos o seguinte resultado que caracteriza $z\left(L_{n}\right)$. Em geral, o teorema seguinte nos dará $z\left(F_{m} \cdot L_{n}\right)$, dadas algumas condições para $m$ e $n$.

Teorema 3.16. Se $m$ e $n$ são inteiros positivos, com $m$ impar, $n>1$ e $\operatorname{mdc}(m, n)=1$, então $z\left(F_{m} \cdot L_{n}\right)=2 m n$. Em particular, $z\left(L_{n}\right)=2 n$, para todo $n>1$.

Demonstração. Por hipótese, $m$ é ímpar. Assim, o Teorema 2.23 b) garante que $L_{n}$ divide $L_{m n}$. Igualmente, a Proposição 2.6 nos informa que $F_{m} \mid F_{m n}$. Desse modo, $F_{m} \cdot L_{n} \mid F_{m n} \cdot L_{m n}=F_{2 m n}$. Portanto, pela Proposição 3.7, z( $\left.F_{m} \cdot L_{n}\right) \mid 2 m n$.

Por outro lado, uma vez que $F_{m} \mid F_{m} \cdot L_{n}$ e $L_{n} \mid F_{m} \cdot L_{n}$, as Proposições 3.5 e 3.6 nos informam, respectivamente, que $m \mid z\left(F_{m} \cdot L_{n}\right)$ e $2 n \mid z\left(F_{m} \cdot L_{n}\right)$. Agora, observe 
que $\operatorname{mdc}(m, n)=1$, por hipótese, e $m$ é ímpar, $\operatorname{logo} \operatorname{mdc}(m, 2 n)=1$, o que nos permite afirmar que $2 m n \mid z\left(F_{m} \cdot L_{n}\right)$.

Dado que $2 m n\left|z\left(F_{m} \cdot L_{n}\right)\right| 2 m n$, obtemos $z\left(F_{m} \cdot L_{n}\right)=2 m n$. Tomando $m=1$, encontramos $z\left(L_{n}\right)=2 n$.

Recentemente, Marques publicou uma série de artigos sobre a ordem de aparição na sequência de Fibonacci. Entre tantos resultados descobertos ele caracterizou $z(n)=n$ e $z(n)=2 n$.

Ele mostrou que $z(n)=n$ se, e somente se, $n=5^{k}$ ou $n=12 \cdot 5^{k}$. E também mostrou que $z(n)=2 n$ se, e somente se, $n=6 \cdot 5^{k}$.

Dessa forma, os únicos números naturais $n$ que têm a ordem de aparição na sequência de Fibonacci igual a $2 n$ são $6,30,150,750, \ldots, 6 \cdot 5^{k}, \ldots$ 


\section{CAPÍTULO 4}

\section{APLICAÇÕES AO ENSINO MÉDIO}

Nesse capítulo, vamos fazer algumas considerações sobre a aplicação da teoria elementar dos números às séries finais do Ensino Médio e propor algumas questões que também podem ser trabalhadas, explorando propriedades algébricas, com alunos do Ensino Fundamental.

O professor interessado pode apresentar aos seus alunos atividades onde eles exercitem a capacidade de conjecturar (isso foi feito, no desenvolvimento do texto, ao observar a Tabela 3.2 e alguns padrões que lá estavam). A partir dessas conjecturas e tendo como ajuda o conhecimento do docente, vários caminhos podem ser trilhados.

Encerrando o trabalho, colocaremos no apêndice uma lista de problemas que, juntamente com os demais tópicos trabalhados, nos capítulos anteriores, podem ser úteis em sala de aula.

\subsection{Pequeno teorema de Fermat}

A primeira consideração importante a ser feita é sobre o pequeno teorema de Fermat (PTF), o qual afirma que $a^{p} \equiv a(\bmod p)$, com $a$ inteiro e $p$ primo. A demonstração dele é acessível ao Ensino Médio e o estudo das congruências e suas aplicações é bastante interessante.

Esse tópico da teoria dos números gera bastante interesse pela simplicidade do seu 
enunciado e pelo poder teórico que ele tem. Defendemos que ensiná-lo é interessante e as possibilidades de trabalho a partir daí são diversas.

Se o professor tiver tempo para trabalhar a definição de congruência e as propriedades que a relação de congruência tem, o PTF "fechará com chave de ouro" essa abordagem sobre a teoria dos números.

Nesse sentido, vamos citar um exemplo de questão cobrada em vestibulares, que aborda conceitos sobre divisibilidade. No primeiro instante, a resolveremos com conceitos trabalhados no Ensino Fundamental e posteriormente a resolveremos usando o PTF.

Exemplo 4.1 (UFMT). Sobre o número natural $n=2^{40}-1$, considere as seguintes afirmativas:

I- n é um múltiplo de 31.

II - n é um múltiplo de 5.

III - n é um número primo.

$I V-n$ é um número par.

Estão corretas as afirmativas:

Primeira solução: (Utilizando conceitos trabalhados no Ensino Fundamental).

Vamos simplesmente fatorar $n$. Lembrando que $a^{2}-b^{2}=(a-b)(a+b)$, com $a, b \in \mathbb{R}$, temos:

$$
\begin{aligned}
n & =2^{40}-1 \\
& =\left(2^{20}\right)^{2}-1=\left(2^{20}-1\right)\left(2^{20}+1\right)=\left(\left(2^{10}\right)^{2}-1\right)\left(2^{20}+1\right) \\
& =\left(2^{10}-1\right)\left(2^{10}+1\right)\left(2^{20}+1\right)=\left(2^{5}-1\right)\left(2^{5}+1\right)\left(2^{10}+1\right)\left(2^{20}+1\right) \\
& =(31)(33)(1025)\left(2^{20}+1\right)=5(31)(33)(205)\left(2^{20}+1\right) .
\end{aligned}
$$

Portanto, as afirmativas corretas são $I$ e $I I$.

Segunda solução: (Utilizando o Pequeno Teorema de Fermat).

Observe, pelo PTF, que $2^{30} \equiv 1(\bmod 31)$. Multiplicando a congruência por $2^{10}$, temos $2^{40} \equiv 2^{10} \equiv 1(\bmod 31)$. Portanto, $31 \mid 2^{40}-1$, ou seja, a afirmação $I$ está correta. 
Analogamente, observe que $2^{4} \equiv 1(\bmod 5)$. Elevando a congruência a 10 , temos $2^{40} \equiv 1(\bmod 5)$, ou seja, $5 \mid 2^{40}-1$. Portanto, a afirmação $I I$ também está correta.

Claramente nas duas soluções $n$ não é primo e também não é par.

O objetivo do exemplo anterior é que o professor, que esteja lendo esse trabalho, se sinta motivado a ir além na sala de aula. Tanto no método da fatoração, como utilizando o PTF, existe o fato interessante de o aluno conseguir descobrir propriedades do número $n=2^{40}-1$ sem o auxílio de uma calculadora. Sem contar o fato de que, em uma calculadora comum, o número $2^{40}-1$ não caberia no visor. Mas, apesar disso, com o suporte que a teoria matemática oferece, o aluno consegue tirar suas próprias conclusões.

Ainda com a ótica sobre o estudo das congruências, observe o segundo exemplo dessa seção.

Exemplo 4.2. Qual o último dígito de $3^{2015}$ ?

Primeira solução: (Observando padrões).

\begin{tabular}{c|c|c|c|c}
\hline Formato do expoente & $4 q$ & $4 q+1$ & $4 q+2$ & $4 q+3$ \\
\hline Último dígito da potência de 3 & 1 & 3 & 9 & 7 \\
\hline
\end{tabular}

Tabela 4.1: Padrão das potências de 3

Tendo em vista que 2015 tem a forma $4 q+3$ segue que $3^{2015}$ termina em 7 .

Segunda solução: (Utilizando o binômio de Newton)

Observe que $3^{2}=10-1$. Elevando a igualdade a 1007, temos:

$$
\begin{aligned}
3^{2014} & =(10-1)^{1007} \\
& =\sum_{k=0}^{1007}\left(\begin{array}{c}
1007 \\
k
\end{array}\right) 10^{k}(-1)^{1007-k} \\
& =10 \cdot A+\left(\begin{array}{c}
1007 \\
0
\end{array}\right) 10^{0}(-1)^{1007} \\
& =10 \cdot A-1
\end{aligned}
$$

Multiplicando por 3 a igualdade $3^{2014}=10 A-1$, obtemos $3^{2015}=10 B-3$.

Portanto, $3^{2015}$ é um múltiplo de 10 menos 3 unidades. Ou seja, termina em 7 . 
Terceira solução: (Utilizando congruências)

Observe que $3^{4} \equiv 1(\bmod 10)$. Elevando a congruência a 503 , temos $\left(3^{4}\right)^{503} \equiv$ $1(\bmod 10)$, ou seja, $3^{2012} \equiv 1(\bmod 10)$. Multiplicando por $3^{3}$, obtemos $3^{2015} \equiv 27 \equiv$ $7(\bmod 10)$. Portanto, o último dígito de $3^{2015}$ é 7.

Em [7], há excelentes questões e exemplos sobre congruências. O leitor interessado deve se esforçar para conhecê-los. Para encerrar essa seção, demonstraremos o Pequeno Teorema de Fermat.

Teorema $4.3(\mathrm{PTF})$. Se $a \in \mathbb{Z}$ e p um número primo, então $a^{p} \equiv a(\bmod p)$.

Demonstração. Se $p=2$, temos $a^{2} \equiv a(\bmod 2)$, pois $a^{2}$ e $a$ têm a mesma paridade.

Suponha $p$ um primo ímpar e, sem perda de generalidade, $a \geq 0$. Vamos usar indução sobre $a$.

O resultado é verdadeiro para $a=0$, pois $p \mid 0$.

Suponha que $a^{p} \equiv a(\bmod p)$, queremos mostrar que $(a+1)^{p} \equiv a+1(\bmod p)$.

Sabemos, pela fórmula do Binômio de Newton, que

$$
(a+1)^{p}=a^{p}+\left(\begin{array}{c}
p \\
1
\end{array}\right) a^{p-1}+\cdots+\left(\begin{array}{c}
p \\
p-1
\end{array}\right) a+\left(\begin{array}{l}
p \\
p
\end{array}\right) .
$$

Subtraindo $(a-1)$ em ambos os lados da igualdade acima, temos:

$$
(a+1)^{p}-(a+1)=a^{p}-a+\left(\begin{array}{l}
p \\
1
\end{array}\right) a^{p-1}+\cdots+\left(\begin{array}{c}
p \\
p-1
\end{array}\right) a .
$$

Pela hipótese de indução, $a^{p}-a$ é divisível por $p$, e sabemos que os números $\left(\begin{array}{l}p \\ i\end{array}\right)$, onde $0<i<p$, são todos múltiplos de $p$. Portanto, $(a+1)^{p} \equiv a+1(\bmod p)$.

\subsection{Sugestão de atividades e problemas}

Para introduzir uma aula sobre sequências numéricas, o professor pode seguir o roteiro abaixo. Essa atividade gera muita curiosidade em sala de aula.

Em primeiro lugar o professor deve pedir para cada aluno da turma individualmente escolher dois números $n_{1}, n_{2}$ quaisquer (podem até ser números reais). Os alunos escolhem esses dois números e não falam pra ninguém, anotando-os no caderno.

A partir daí, trabalhando a ideia de sequências recursivas, o professor pede que eles escrevam os outros números, a partir do terceiro número $-n_{3}-$ como soma dos dois 
números anteriores, parando no décimo. Ou seja, $n_{3}=n_{2}+n_{1}, n_{4}=n_{3}+n_{2}, \ldots, n_{10}=$ $n_{9}+n_{8}$.

Nesse instante o professor lança o desafio, dizendo que é capaz de saber o valor da soma $\left(S_{10}=n_{1}+n_{2}+\cdots+n_{10}\right)$ dos 10 números de algum aluno, sem conhecê-los, antes que o próprio aluno o faça.

Note que, dessa forma, o professor nunca conseguiria descobrir o valor da soma. Mas essa afirmação do professor deixa a turma curiosa.

Para conseguir descobrir o valor $S_{10}$, ele (o professor) precisa saber apenas o valor do sétimo número $n_{7}$. E para dar prosseguimento ao desafio, pede que algum aluno informe somente o valor de $n_{7}$ e com isso descobrirá o valor da soma $S_{10}$ antes mesmo que o próprio aluno consiga efetuar o cálculo.

Uma vez que o aluno deve provavelmente somar os 10 valores e o professor só irá efetuar uma multiplicação por 11 , ou seja $11 n_{7}$ (o que é muito simples), inevitavelmente a turma ficará impressionada com a rapidez do professor.

Posteriormente a essa breve explanação o professor deverá instigar os alunos a descobrirem o porquê do truque funcionar.

O que foi dito acima pode ser resumido no problema seguinte.

Exemplo 4.4. A soma de 10 números consecutivos de uma sequência recursiva como a de Fibonacci, respectivamente sequência dos números de Lucas, é igual a 11 vezes o sétimo número entre os 10 listados.

Solução. Sejam $n_{1}=a$ e $n_{2}=b$ dois números consecutivos de uma das referidas sequências. Dessa forma,

$$
\begin{aligned}
& n_{1}=a \\
& n_{2}=b \\
& n_{3}=a+b \\
& n_{4}=a+2 b \\
& n_{5}=2 a+3 b \\
& n_{6}=3 a+5 b \\
& n_{7}=5 a+8 b \\
& n_{8}=8 a+13 b \\
& n_{9}=13 a+21 b \\
& n_{10}=21 a+34 b .
\end{aligned}
$$


Assim a soma $S_{10}=n_{1}+\cdots+n_{10}$ dos 10 números consecutivos é $S_{10}=55 a+88 b=$ $11(5 a+8 b)=11 n_{7}$. O que mostra o porquê do truque funcionar.

Para motivar a interdisciplinaridade entre conceitos matemáticos o professor pode usar o exemplo a seguir.

Observando os números $\Phi=(1+\sqrt{5}) / 2, \Phi-1=\phi$ e construindo um paralelepípedo com medidas $1, \phi$ e $\Phi$, conhecido como tijolo de Fibonacci, encontramos uma relação interessante entre os números irracionais $\pi$ e $\Phi$ ao inscrever esse sólido em uma esfera de raio 1.

Exemplo 4.5. Mostre que a razão entre a área da esfera que circunscreve o tijolo de Fibonacci e a área do tijolo é igual a $\pi / \Phi$.

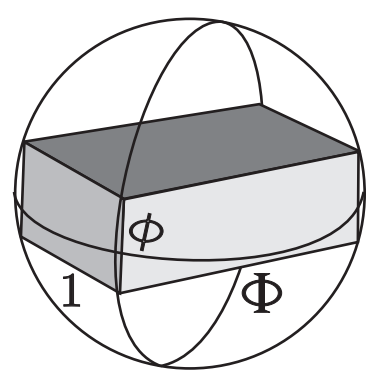

Figura 4.1: Tijolo de Fibonacci

Solução. De fato, o raio $R$ da esfera é igual à metade da diagonal do paralelepípedo. Calculando esse valor, temos:

$$
\begin{aligned}
R & =\frac{\sqrt{\Phi^{2}+\phi^{2}+1}}{2} \\
& =\frac{\sqrt{\Phi^{2}+(\Phi-1)^{2}+1}}{2} \\
& =\frac{\sqrt{2 \Phi^{2}-2 \Phi+2}}{2} \\
& =\frac{\sqrt{2\left(\Phi^{2}-\Phi+1\right)}}{2}=1 .
\end{aligned}
$$

Como o raio $R$ da esfera é igual a 1 , a área da esfera é igual a $4 \pi$. Observe que a área do tijolo de Fibonacci é dada por $2(\Phi \phi+\Phi+\phi)=2(1+\sqrt{5})$. Assim,

$$
\frac{\mathrm{S}_{\text {Esfera }}}{\mathrm{S}_{\text {Tijolo }}}=\frac{4 \pi}{2(1+\sqrt{5})}=\frac{2 \pi}{1+\sqrt{5}}=\frac{\pi}{\frac{1+\sqrt{5}}{2}}=\frac{\pi}{\Phi} .
$$


Por último, para poder trabalhar a ideia da demonstração por absurdo e para mostrar, de maneira simples, a beleza da teoria dos números, vamos "mostrar" que todos os números naturais são notáveis. A nossa motivação será um trecho de um livro (ver [22]) que transcreveremos a seguir.

"Certa vez, o grande matemático inglês Hardy foi visitar, em Londres, o célebre matemático indiano Ramanujan. Ao chegar Hardy comentou que o táxi em que viera ostentava um número completamente desinteressante, a saber, 1729. Ramanujan imediatamente replicou: 'Mas como assim, desinteressante? O número 1729 é o menor natural que pode ser escrito de duas maneiras diferentes como soma de dois cubos.' De fato, $1729=1^{3}+12^{3}=9^{3}+10^{3}$."

Exemplo 4.6. Todo número natural é notável.

"Demonstração."

Suponha que exista um conjunto de números naturais não notáveis. Esse conjunto, necessariamente possui um elemento mínimo, não notável $n$, pelo princípio da boa ordenação (PBO). Logo esse elemento possui uma característica especial entre todos os outros, passando a ser interessantíssimo. Mas isto é uma contradição, pois o conjunto formado, por hipótese, era de números não notáveis. Portanto $n$ é notável!

As considerações feitas no trabalho não esgotam as discussões sobre a aplicação de novos conceitos aos estudantes. E também, de nenhuma forma, elas são a melhor maneira para se trabalhar algum conteúdo. Elas servem como exemplo para quem tiver interesse no assunto.

Em geral, a maior esperança com o que foi apresentado é que o leitor tenha a mesma dedicação pela melhoria da educação no Brasil como o estudante que escreveu esse texto.

No apêndice A estão algumas questões que podem ser trabalhadas no Ensino Médio ou Ensino Fundamental. 


\section{CONSIDERAÇÕES FINAIS}

O grande diferencial desse texto pode ser percebido no Capítulo 3. Nele é abordado um assunto completamente fora da matriz curricular do Ensino Médio, porém as demonstrações são feitas tendo como base conceitos de divisibilidade que podem ser aprofundados perfeitamente por alunos que estejam dispostos a estudar Matemática.

É importante destacar que o conhecimento abstrato, utilizado nas demonstrações matemáticas, desperta no aluno uma capacidade de análise crítica e de argumentação consistente. Isso ajuda em todos os campos da formação do saber.

Em um contexto da educação em que se pergunta qual o papel da Matemática na vida social, enxergá-la como reflexo do desenvolvimento humano nos permite entender que a busca pelo novo é incessante. De tal forma que a cada dia o professor deve se motivar para o trabalho e com isso ser luz para a vida dos jovens em formação.

Essa pesquisa me propiciou isso. Tanto pude vivenciar a Matemática como um celeiro de descobertas, como pude ver crescer novos conhecimentos, naqueles alunos que tive, durante a realização desse mestrado profissionalizante. Termino o curso com a sensação de um dever cumprido e com a esperança de poder contribuir socialmente, focado na melhoria da educação.

Posso afirmar que, sem sombras de dúvidas, fui orientado por um gigante da Matemática! Um professor que faz despertar no aluno a vontade de estudar. Que mesmo estando envolvido com pesquisas de ponta, publicando artigos nas revistas mais conceituadas do mundo, reconhece a real necessidade da participação na formação de professores! Após esse mestrado percebi verdadeiramente o significado da frase 
de William Arthur Ward: "O professor medícre conta. O bom professor explica. O professor superior demonstra. O grande professor inspira."

Que todos nós envolvidos de qualquer maneira com a educação consigamos, em um esforço conjunto, colocar a Matemática brasileira em um lugar de destaque.

Espero com esse trabalho contribuir para a melhoria da formação de professores e alunos. Espero que qualquer pessoa que o tenha lido se sinta tão motivado a ir além, como me senti durante a sua construção. Deparando-me com tantas informações inéditas consegui perceber que a Matemática ainda está se desenvolvendo e me senti motivado a fazer parte desse processo.

É muito desafiador, por exemplo, o fato de saber se há infinitos primos na sequência de Fibonacci. É bastante curioso e instigante pensar em um problema que ainda não foi solucionado. E após a solução desse referido problema acompanhar outros novos que surgem.

Em relação à ordem de aparição, aonde uma fórmula fechada para $z(n)$ dado qualquer número natural $n$ nos levaria? Será que é possível demonstrar que não existe tal fórmula para $z(n)$ ?

Enfim, o sentimento após esse mestrado é de uma cachoeira de conhecimentos derramada sobre a minha cabeça, mesmo sabendo que essa cachoeira não era nada mais do que uma pequena gota de informação. 


\section{APÊNDICE A}

\section{PROBLEMAS APLICÁVEIS AO ENSINO}

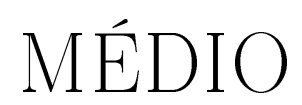

Problema 1 (EUA). A Sequência de Fibonacci $1,1,2,3,5,8,13,21, \ldots$ começa com dois 1 s e cada termo seguinte é a soma de seus dois antecessores. Qual dos dez dígitos (do sistema de numeração decimal) é o último a aparecer na posição das unidades na sequência de Fibonacci?

Problema 2. Seja $\alpha$ a maior raiz de $x^{2}-x-1=0$. Determine o valor de $\alpha^{5}-5 \alpha$.

Problema 3. Há 10 lâmpadas enfileiradas em uma sala. Quantas configurações existem se não puder haver duas lâmpadas adjacentes ligadas simultaneamente?

Problema 4. Escreva o número natural $m=2015$ como uma soma finita de números de Fibonacci distintos e não consecutivos.

Problema 5 (UnB - 1/2009, adaptado). A razão áurea é uma relação matemática definida algebricamente pela expressão $\frac{a+b}{a}=\frac{a}{b}=\varphi$, em que a e b representam números reais, e $\varphi$, uma constante de valor aproximado igual a 1,618.

A partir da definição algébrica da razão áurea, mostre que $\varphi$ é uma das soluções da equação de segundo grau $\varphi^{2}=\varphi+1$.

Problema 6. Mostre que $\left(\begin{array}{c}10 \\ 0\end{array}\right)+\left(\begin{array}{l}9 \\ 1\end{array}\right)+\left(\begin{array}{l}8 \\ 2\end{array}\right)+\left(\begin{array}{l}7 \\ 3\end{array}\right)+\left(\begin{array}{l}6 \\ 4\end{array}\right)+\left(\begin{array}{l}5 \\ 5\end{array}\right)$ é um número de Fibonacci. 
Problema 7. Seja $L_{n}=\alpha^{n}+\beta^{n}$ o n-ésimo número de Lucas, com $\alpha=(1+\sqrt{5}) / 2$ e $\beta=(1-\sqrt{5}) / 2$, tal que $L_{n+2}=L_{n+1}+L_{n}$, com $L_{0}=2$ e $L_{1}=1$. Mostre que $L_{n} L_{n+1}=L_{2 n+1}+(-1)^{n}$.

Problema 8. Mostre que é válida a identidade $\left(\frac{L_{n}+\sqrt{5} F_{n}}{2}\right)^{k}=\frac{L_{n k}+\sqrt{5} F_{n k}}{2}$, onde $L_{n}=$ $\alpha^{n}+\beta^{n}$ e $F_{n}=\left(\alpha^{n}-\beta^{n}\right) / \sqrt{5}$ denotam, respectivamente, o n-ésimo número de Lucas e o n-ésimo número de Fibonacci, com $\alpha=(1+\sqrt{5}) / 2, \beta=(1-\sqrt{5}) / 2$ e $k$ é um inteiro não negativo.

Problema 9. Mostre que $F_{2 n}=F_{n} \cdot L_{n}$ para todo $n$ inteiro não negativo, onde $L_{n}=$ $\alpha^{n}+\beta^{n}$ e $F_{n}=\left(\alpha^{n}-\beta^{n}\right) / \sqrt{5}$ denotam, respectivamente, o n-ésimo número de Lucas e o n-ésimo número de Fibonacci.

Problema 10. Mostre que para quaisquer inteiros não negativos a e b, temos $F_{a} L_{b}=$ $F_{a+b}+(-1)^{b} F_{a-b}$, onde $L_{n}=\alpha^{n}+\beta^{n}$ e $F_{n}=\left(\alpha^{n}-\beta^{n}\right) / \sqrt{5}$ denotam, respectivamente, o n-ésimo número de Lucas e o n-ésimo número de Fibonacci.

Problema 11. Sabendo que a soma dos $n$ primeiros números de Fibonacci é igual a $F_{n+2}-1$, mostre que a soma de $2 n$ números consecutivos da sequência de Fibonacci é divisivel por $F_{n}$, para todo $n$ par.

Problema 12. Mostre que a soma de dois quadrados de números de Fibonacci consecutivos é um número de Fibonacci, ou seja, $F_{n-1}^{2}+F_{n}^{2}=F_{2 n-1}$ para todo $n$ natural.

Problema 13 (UnB - 1/2012, adaptado). Julgue a afirmação seguinte.

Seja $F_{n}$ o n-ésimo número de Fibonacci. O sistema linear homogêneo cuja matriz dos coeficientes é a matriz A, apresentada a seguir, tem solução única.

$$
A=\left(\begin{array}{cccc}
F_{1} & F_{2} & F_{3} & F_{4} \\
F_{5} & F_{6} & F_{7} & F_{8} \\
F_{9} & F_{10} & F_{11} & F_{12} \\
F_{13} & F_{14} & F_{15} & F_{16}
\end{array}\right) .
$$

Problema 14. Seja $n$ um número natural e $F_{m}$ o $m$-ésimo número de Fibonacci. A ordem de aparição de $n$ na sequência de Fibonacci, denotada por z(n), é o menor inteiro positivo $k$ tal que $n \mid F_{k}$. Calcule $z(n)$ para $n \in\{1,2,3, \ldots, 11,12\}$.

Problema 15 (UnB - 1/2012, adaptado). Seja $F_{n}$ o n-ésimo número de Fibonacci. Mostre que se $x$ é um número real tal que $\left|x-\frac{F_{7}}{F_{6}}\right|>2$, então $x>2$ ou $x<-0,3$. 
Problema 16 (UnB - 1/2012, adaptado). Mostre que se $\alpha$ e $\beta$ são as raízes positiva e negativa, respectivamente, do polinômio $f(x)=x^{2}-x-1$, então $\alpha^{3}-\beta^{3}=\sqrt{5} F_{3}$.

Problema 17 (IME 2007/2008, adaptado). Uma série de Fibonacci é uma sequência de valores definida da seguinte maneira: Os dois primeiros termos são iguais à unidade, ou seja, $F_{1}=F_{2}=1$ e cada termo, a partir do terceiro, é igual à soma dos dois termos anteriores, isto é: $F_{n}=F_{n-1}+F_{n-2}$. Se $F_{18}=2584$ e $F_{21}=10946$ então $F_{22}$ é igual $a:$

(A) 12225

(B) 13530

(C) 17711

(D) 20412

(E) 22121

Problema 18. Mostre que a razão entre a área da esfera que circunscreve o tijolo de Fibonacci, na figura abaixo, (paralelepipedo de lados $1, \Phi=(1+\sqrt{5}) / 2$ e $\phi=(\sqrt{5}-1) / 2)$ e a área do tijolo é igual a $\pi / \Phi$.

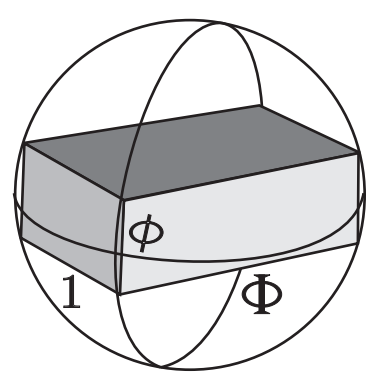

Figura A.1: Esfera circunscrita ao tijolo de Fibonacci

Problema 19 (IME 1997/1998). Considere a sequência cujos primeiros termos são: $1,2,3,5,8,13,21,34,55, \ldots$ Seja $a_{n}$ seu n-ésimo termo. Mostre que $a_{n}<((1+\sqrt{5}) / 2)^{n}$ para todo $n \geq 2$.

Problema 20. Mostre que a soma de 10 números consecutivos da sequência de Fibonacci, respectivamente sequência dos números de Lucas, é igual a 11 vezes o sétimo número entre os 10 listados. 
Problema 21. Mostre que $L_{n}=F_{n+1}+F_{n-1}$ para todo $n$ natural. Onde $F_{n}$ e $L_{n}$ denotam respectivamente o n-ésimo número de Fibonacci e o n-ésimo número de Lucas.

Problema 22. Mostre que

$$
\left[\begin{array}{ll}
1 & 1 \\
1 & 0
\end{array}\right]^{n}=\left[\begin{array}{cc}
F_{n+1} & F_{n} \\
F_{n} & F_{n-1}
\end{array}\right]
$$

Posteriormente, calculando o determinante de ambos os lados da igualdade, deduza a relação de Cassini: $(-1)^{n}=F_{n-1} F_{n+1}-F_{n}^{2}$.

Problema 23. Considere $F_{n}$ a sequência de Fibonacci. Mostre que $F_{n}<\left(\frac{7}{4}\right)^{n}$.

Problema 24. Mostre que a razão entre uma diagonal qualquer de um pentágono regular e um de seus lados é igual a $\alpha=(1+\sqrt{5}) / 2$.

Problema 25. Prove que $\alpha^{n}=\alpha F_{n}+F_{n-1}$, onde $\alpha=(1+\sqrt{5}) / 2$ e $F_{n}$ denota o n-ésimo número de Fibonacci.

Problema 26. Para quais $n \in \mathbb{N}$, o número $\alpha^{n}-n \alpha$ é inteiro?

Problema 27. Prove que $\alpha^{n}=\alpha^{n-1}+\alpha^{n-2}$, onde $\alpha=(1+\sqrt{5}) / 2$.

Problema 28. Seja $M=\left(\begin{array}{ccc}F_{3 n+1} & F_{3 n+3} & F_{3 n+6} \\ F_{3 n} & F_{3 n+2} & F_{3 n+5} \\ F_{3 n+3} & F_{3 n+9} & F_{3 n+4}\end{array}\right)$. Mostre que não existe $n \in \mathbb{N}$ tal que $\operatorname{det} M=0$.

Problema 29. Mostre que:
i) $2 F_{m+n}=F_{m} L_{n}+F_{n} L_{m}$;
ii) $2 L_{m+n}=L_{m} L_{n}+5 F_{m} F_{n}$;
iii) $L_{m+n}=L_{m} L_{n}-(-1)^{n} L_{m-n}$;
iv) $L_{n}^{2}=L_{n-1} L_{n+1}+5(-1)^{n}$;
v) $L_{n}^{2}=5 F_{n}^{2}+4(-1)^{n}$;
vi) $5 F_{n}=L_{n-1}+L_{n+1}$. 


\section{REFERÊNCIAS BIBLIOGRÁFICAS}

[1] AlEnCAR, F. E., Funções Aritméticas Números Notáveis, Nobel, (1988).

[2] ALEX, B., Alex no País dos Números: Uma viagem ao mundo maravilhoso da matemática, Companhia das Letras (2012).

[3] BICKNELL, M. And HOGGATT, V.E. JR., A primer for the Fibonacci numbers: Part IX, The Fibonacci Quarterly, 9.5, (1971), pp. 529-536.

[4] CARMICHAEL, R. D., On the Numerical Factors of the Arithmetic Forms $\alpha^{n} \pm$ $\beta^{n}$, Annals of Mathematics, vol. 15, (1913), pp. 30-48.

[5] CONTADOR, P. R. M., A matemática na arte e na vida, Livraria da Física (2011).

[6] HAN J. S., KIM AND NEGGERS, J., The Fibonacci-norm of a positive integer: observations and conjectures, International Journal of Number Theory, World Scientific, (2010).

[7] HEFEZ A., Aritmética, Coleção PROFMAT, SBM, (2013).

[8] KOSHY T., Fibonacci and Lucas numbers with Applications, Wiley, New York, (2001).

[9] LENGYEL T., The order of the Fibonacci and Lucas numbers, The Fibonacci Quarterly, 33.3, (1995), pp. 234-239. 
[10] LIMA, E. L., Análise Real Funções de Uma Variável, Coleção Matemática Universitária, SBM (2007), pp. 17-18.

[11] LIMA, et al, A Matemática do Ensino Médio, Coleção do professor de Matemática, SBM, Vol. 2 (1998), pp. 74-76.

[12] LUCA, F. E OYONO, R., An exponential Diophantine equation related to powers of two consecutive Fibonacci nunbers, Proceedings of the Japan Academy, Vol. 87, Ser. A., (2011), pp. 45-50.

[13] LUCA, F. E POMERANCE, C., On the local behavior of the order of appearance in the Fibonacci sequence, (2013).

[14] MARQUES, D. E TOGBÉ, A., On the sum of powers of two consecutive Fibonacci numbers, Proceedings of the Japan Academy, Vol. 86, Ser. A., (2010), pp. 174-176.

[15] MARQUES, D., On Integer Numbers with Locally Smallest Order of Appearance in the Fibonacci Sequence, International Journal of Mathematics and Mathematical Sciences, (2011), pp. 1-4.

[16] MARQUES, D., Teoria dos Números Transcendentes, Textos Universitários, SBM, (2013).

[17] MARqUES, D., Sharper Upper Bounds for the Order of Appearance in the Fibonacci Sequence, The Fibonacci Quarterly, 51.3, (2013), pp. 233-238.

[18] MARTINEZ, F. B. et al, Teoria dos números: um passeio com primos e outros números familiares pelo mundo inteiro, Projeto Euclides, IMPA, (2013).

[19] MARTineZ, F. B. et al, Tópicos de Teoria dos Números, Coleção PROFMAT, SBM (2012), pp. 114-124.

[20] SALLÉ, H. J. A., A maximum value for the rank of apparition of integers in recursive sequences, The Fibonacci Quarterly, 13.2, (1975), pp. 159-161.

[21] SANTOS, J. P., Introdução à Teoria dos números, Coleção Matemática Universitária, IMPA, (2012).

[22] STEWUART, I., Almanaque das curiosidades matemáticas, Pelican, (2008). 
[23] www.fq.math.ca

[24] http://www.maths.surrey.ac.uk/hosted-sites/R.Knott/Fibonacci/fibtable.html, acessado em 06/07/2015 às 04:08. 